Document downloaded from:

http://hdl.handle.net/10251/65308

This paper must be cited as:

Salvador Rubio, FJ.; Carreres Talens, M.; Jaramillo Císcar, D.; Martínez López, J. (2015). Analysis of the combined effect of hydrogrinding process and inclination angle on hydraulic performance of diesel injection nozzles. Energy Conversion and Management. 105:13521365. doi:10.1016/j.enconman.2015.08.035.

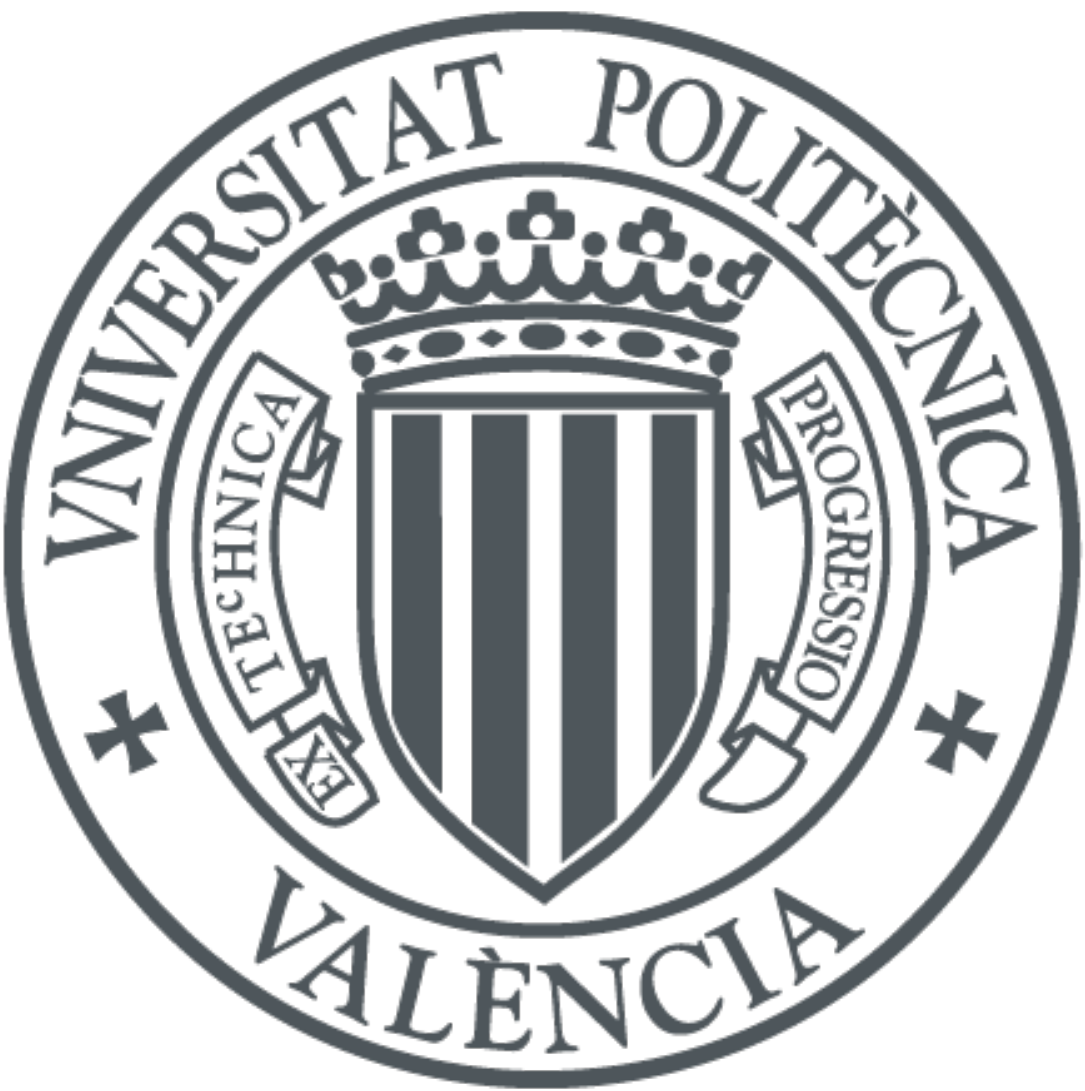

The final publication is available at

http://dx.doi.org/10.1016/j.enconman.2015.08.035

Copyright Elsevier

Additional Information 
ANALYSIS OF THE COMBINED EFFECT OF HYDROGRIDING PROCESS AND INCLINATION ANGLE ON HYDRAULIC PERFORMANCE OF DIESEL INJECTION NOZZLES.

Salvador, FJ $\left.{ }^{1}{ }^{*}\right)$, Carreres, M. ${ }^{1}$, Jaramillo, D. ${ }^{1}$, Martínez-López, J. ${ }^{2}$

${ }^{1}$ CMT-Motores Térmicos, Universitat Politècnica de València

Camino de Vera s/n, E-46022, Valencia, Spain.

${ }^{2}$ Koenigsegg Automotive AB

26274 Ängelholm, Sweden

(*) Corresponding author:

Dr. F. Javier Salvador, fsalvado@ mot.upv.es

CMT-Motores Térmicos, Universitat Politècnica de València

Camino de Vera s/n, E-46022 Spain.

Telephone: +34-963879659

FAX: +34-963877659 


\begin{abstract}
A computational study to investigate the influence of the orifices inclination and the rounding radius at the orifice inlet (consequence of the hydro-erosive grinding process applied after the orifices machining) over the internal nozzle flow is performed in this paper. The study starts with the analysis of experimental results where the mass flow and momentum flux of two nozzles with very different values of these two variables are compared. This analysis shows relatively small differences in terms of mass flow and momentum flux, since the higher losses associated to the higher deflection of the streamlines with a higher inclination of the orifices are counteracted by the higher rounding radius, which favors the flow entrance to the orifice.
\end{abstract}

To explain this experimental outcome, an extensive computational study involving nine geometries that combine different inclination angles and rounding radius is conducted, in order to quantify the influence of both parameters on the flow separately, as well as to assess the potential of their combination. These geometries are compared in terms of discharge coefficient, critical cavitation conditions and effective injection velocity, among others. Results show differences up to $15 \%$ in terms of mass flow rate and $8 \%$ for the effective injection velocity among the two extreme cases (lowest inclination and highest hydro-erosion level versus the nozzle with the highest inclination and lowest hydro-erosion level). Given the importance of these phenomena on the subsequent mixing and combustion processes, the results hereby presented are of interest for Diesel engines injectors and combustion chambers designers.

\title{
KEYWORDS
}

diesel, injection, nozzle, inclination, hydrogrinding, cavitation.

\section{LIST OF NOTATION}

$A_{\text {eff }}$ outlet effective area 

$A_{o} \quad$ outlet area
$C_{a}$ area coefficient
$C_{d}$ discharge coefficient
$C_{v} \quad$ velocity coefficient
$C_{\varepsilon 1}$ constant for $\varepsilon$ transport equation calculation
$C_{\varepsilon 2}^{o} \quad$ variable for $\varepsilon$ transport equation calculation
$C_{\varepsilon 2}$ constant for $\varepsilon$ transport equation calculation
$C_{\mu} \quad$ constant for turbulent viscosity calculation
c speed of sound

$D_{i} \quad$ diameter at the orifice inlet

$D_{o}$ diameter at the orifice outlet

$K$ cavitation number

$k \quad$ turbulent kinetic energy

$L \quad$ orifice length

$\dot{M}_{f} \quad$ momentum flux

$\dot{m}_{f}$ mass flow

$P \quad$ pressure

$P_{\text {back }}$ discharge back pressure 
$P_{i n j} \quad$ injection pressure

$P_{\text {vap }}$ vaporisation pressure

$p_{k} \quad$ production of turbulent kinetic energy

$r \quad$ rounding radius at the inlet orifice

$t$ time

$u \quad$ velocity

$\bar{u} \quad$ averaged velocity

$u^{\prime} \quad$ fluctuating velocity

$u_{\text {eff }}$ effective velocity

$u_{t h} \quad$ theoretical velocity

$S \quad$ mean strain

$S_{i j} \quad$ strain tensor

\section{GREEK SYMBOLS:}

$\Delta P$ pressure drop, $\Delta P=P_{\text {in }}-P_{b}$

$\Psi \quad$ fluid compressibility

$\Psi_{l} \quad$ liquid compressibility

$\Psi_{v} \quad$ vapour compressibility

$\alpha_{\varepsilon} \quad$ constant for $\varepsilon$ transport equation calculation 
$\alpha_{\mathrm{k}} \quad$ constant for $k$ transport equation calculation

$\beta \quad$ constant for the turbulence model

$\gamma \quad$ vapour mass fraction

$\varepsilon \quad$ turbulence dissipation rate

$\mu \quad$ fluid viscosity

$\mu_{l} \quad$ liquid viscosity

$\mu_{T} \quad$ turbulent viscosity

$\mu_{v} \quad$ vapour viscosity

$\eta \quad$ expansion parameter

$\eta_{0} \quad$ constant for the turbulence model

$\rho \quad$ fluid density

$\rho_{l} \quad$ liquid density

$\rho_{l, \text { sat }}$ liquid density at saturation

$\rho_{l}{ }^{o} \quad$ liquid density at a given temperature condition

$\rho_{v, \text { sat }}$ vapour density at saturation

$\rho_{v} \quad$ vapour density

CA nozzle orifice angle

\section{INTRODUCTION}


It is well known that the air-fuel mixing process in diesel engines has a strong influence on the combustion efficiency and its emissions. The fuel is injected into the combustion chamber as a spray and mixes with the surrounding air for several milliseconds. The efficiency of this mixing process depends mainly on the injection pressure and the geometrical characteristics of the injector nozzle ([1], [2], [3]). The particularities of the diesel combustion lead to the use of nozzles with diameters below $200 \mu \mathrm{m}$ and injection pressures up to 2500 bar in order to achieve high velocities of the fuel at the nozzle outlet. These three factors (small diameters, high pressure and high velocities) make difficult the analysis and the understanding of the internal flow. Its study becomes even more difficult when cavitation phenomenon occurs due to the presence of two-phase flow and its strong impact on the spray formation $([4][5][6][7][8])$.

There are many experimental studies published in the literature about the internal flow in diesel injection nozzles. In the first works, the authors focused on the influence of the nozzle geometry on the discharge coefficient ([9]). More recently, advance optical techniques have been used to characterize the cavitation development in transparent orifices ([10]) and transparent nozzles ([11][12]). Another strategy followed by many researchers to get an insight into the internal flow at cavitating conditions has been the use of spray momentum flux measurements ([13][14][15]).

Besides experimental works, the cavitation phenomenon in diesel injector nozzles has been also studied with CFD tools thanks to the development of complex models based on different hypothesis and assumptions. For example, Alajbegovic et al. [16] developed a two-phase flow approach based on the introduction of certain number of bubbles that could grow depending on the pressure. The grow of each bubble was calculated with the Rayleigh-Plesset equation. Andriotis et al. [17] developed a similar bubble model although the bubbles were divided in small groups instead of been calculated individually. A completely different approach was developed by Schmidt et al. [18], who modeled the internal flow as a homogeneous mixture of liquid and vapour with intermediate 
properties between the pure liquid and pure vapour phases. The properties of this mixture were calculated as a function of the vapour fraction, which was determined by means of a barotropic equation of state. This model showed a great stability for high speed flows in comparison with those models based on the Rayleigh-Plesset equation and has led to several versions of this model applied for the study of cavitating flows in diesel injectors ([8][19][20][21][22][23]).

Discharge coefficient and cavitation inception depend on nozzle geometry and injection pressure conditions, or more generally, the Reynolds number ([6][24]) of the flow. If a nozzle with cylindrical orifices, and so, prone to cavitate ([24]), is considered, two of the most influential parameters on cavitation inception are the rounding radius at the orifice inlet and the orifice angle, or angle formed by the hole axis and the injector axis. Both parameters are shown in Figure 1.

The rounding radius at the orifice entrance is a consequence of the hydro-erosive grinding process to which the nozzles are subjected after their production. This process is carried out using an abrasive liquid with the aim of rounding the sharp edges at the orifice inlet, and thus, increasing the discharge coefficient [25]. In fact, when the injector is running, the flow between the needle and its seat experiences a deflection when it enters to the orifice, which produces an important pressure loss. The higher the level of rounding, the smaller the pressure loss and therefore the higher the discharge coefficient. Furthermore, the increment of the rounding radius also makes the nozzle less prone to cavitate as established in [24]. The hydro-grinding intensity is defined as a percentage that indicates the increase in mass flow rate obtained with regard to the initial situation before applying the process. Thus, we can find levels of hydro-erosive grinding of 5\%, 10\%, 15\% ...[25].

As far as the orifice angle is concerned, it seems obvious that its increment entails an increase in the tendency of cavitation due to the higher deflection that the flow suffers as the angle becomes bigger [24]. 
Nevertheless, the influence of both parameters on flow properties in terms of cavitation or discharge coefficient should not be studied independently but interrelated. In fact, in general terms, higher orifice angles are related to higher rounding radius because of the higher capacity of the abrasive fluid for eroding the orifice inlet. On the other hand, after the hydro-erosive grinding process, the value of the rounding radius is not known even for manufacturers. Nevertheless, non-intrusive techniques such as silicone molding [6] and X-Rays [26] can be used in order to precisely determine the internal geometry of the nozzles.

As far as the inclination angle is concerned, its value depends on the engine configuration and the optimal value pursues the maximum heterogeneity of fuel in the combustion chamber. A clear distinction should be made between a four-valve cylinder engine configuration and a two-valve cylinder engine configuration. On the former, the injector is located in the cylinder axis totally vertical and centred. In this case, the inclination angle of all the orifices is the same and its value depends on the combustion chamber geometry. On the latter, the injector is assembled with a certain inclination angle in relation to the cylinder axis, and as a consequence, all the orifices have a different inclination.

There are some studies in the literature about the influence of orifice inclination or rounding radius on flow features ([22], [25], [27]), but these geometrical characteristics are not dealt with jointly in these publications.

In addition, in some of them, such as in La Forgia, et al. [27], due to their antiquity and limitations of the code employed, the cavitation phenomenon and its consequences are not analysed correctly. In others, like de la Morena et al. [22], although an appropriate code is used for the study of cavitation, only the orifice inclination is considered without taking into account the linkage that it may have with the rounding radius of the nozzle orifices. 
In the present study, the influence of both parameters is analysed jointly, in such a way that it will be possible to quantify the influence on the flow of each of them separately, as well as determining which of their combinations leads to the most favourable flow conditions (higher discharge coefficient and injection velocity) and which lead to less favourable flow conditions (lower discharge coefficient and injection velocity). In the end, the idea is to also quantify the differences in terms of mass flow and effective injection velocity among real nozzles depending on their different inclinations or rounding radius at the inlet.

The objective of the present research work focuses on the influence of the orifice inclination and the inlet rounding radius on the characteristics of the internal flow in diesel injection nozzles. The performance of each nozzle configuration has been evaluated by analysing its permeability in terms of maximum mass flow rate across the hole, and the maximum momentum flux available at the nozzle outlet, which are the most representative hydraulic characterization parameters in the frame of diesel injection systems [13].

The study consists of two different parts: the experimental one and the computational one. In the first part, two nozzles of six orifices with the same diameter and different inclination angles and inlet rounding radius are characterised. These nozzles are tested in terms of mass flow and momentum flux. In order to try to give an explanation to the results, the authors have performed an extensive computational study in which, besides reproducing the results of the previous nozzles (which also served as the code validation), seven additional nozzles have been simulated in order to explore and analyse in more detail the influence of both geometrical parameters on the flow. Results show that the nozzle orifices with a higher inclination with respect to the injector axis and lower rounding radius exhibit a worse behaviour than those with a lower inclination and higher rounding radius. Moreover, the first ones are more prone to cavitate, whereas the second ones are less susceptible to do so, also showing a change in the cavitation pattern with respect to the first ones. In addition, a remarkable 
result is the fact that the differences in terms of mass flow rate may even reach $15 \%$ when comparing the extreme cases. For these same cases, the differences in terms of effective injection velocity are up to an $8 \%$. Given the importance of these phenomena on the subsequent mixing and combustion processes, these results are of interest for Diesel engines injectors and combustion chambers designers. In the study, experimental facilities such as a mass flow rate meter and a momentum test rig able to measure the momentum flux at the orifice outlet are used to characterise 2 of the 9 nozzles considered in the computational part. For the numerical study, a Homogeneous Equilibrium Model able to model the cavitation phenomenon was used. The code is implemented in the version 1.5 of OpenFOAM [28]. Although the code has been previously validated in an extensive way with several nozzles and calibrated orifices $([8][13][20][21])$, it is also validated in the present work against the experimental results of two of the nozzles considered in this study. The validation has been made in terms of mass flow measurements, momentum flux measurements and injection velocity at the nozzle outlet.

As far as the structure of the paper is concerned, it has been divided into 6 sections.

First of all, in section 2, a description of the experimental facilities used for the characterization of the two nozzles is given. This characterization is useful for extracting the first conclusions about the influence of inclination and the inlet radius, as well as for validation purposes of the CFD approach used for the computational part of the study. In section 3, the CFD approach description is made, where the equations governing the model and how they are solved are explained in detail. Following, the geometry characteristics of the nozzles involved in the study and the calculation set-up used for the simulations are described in section 4 . In section 5 the results of mass flow and momentum flux of the two nozzles available for experimental characterization are presented. These results are compared to the computational ones in order to quantify the error committed in predicting the results that are displayed and analysed in section 6, where seven additional nozzles are simulated and 
compared with the aim of shedding light on the influence of these geometrical parameters on the flow pattern and characteristics. Finally, the main conclusions of the investigation are drawn in section 7.

\section{EXPERIMENTAL FACILITIES USED FOR NOZZLES CHARACTERIZATION.}

\subsection{Injection Rate Meter}

All the nozzles analyzed in the present paper have been characterized with an Injection Rate Meter. This equipment is based on the Bosch method [29], and its operating mode consists of the fuel injection in a long tube previously filled with diesel fuel. The fuel discharge through the injector leads to a pressure rise in the tube which is proportional to the injected mass flow [30]. Thus, it is possible to get the injection rate from the pressure evolution over time with an uncertainty below $1.5 \%$.

\subsection{Spray Momentum test Rig}

Figure 2 shows the equipment used to measure the momentum flux of a diesel spray whose uncertainty is around $1.8 \%$. The most important parts of this test rig are the steel chamber, the pressure sensor, the optical access and the inlet/outlet valves.

The main body of the test rig consists of a cylindrical steel chamber which can be pressurized up to $10 \mathrm{MPa}$. This feature, together with the possibility of adjusting the injection pressure and the injection length allow the study of the spray behavior in similar conditions to those existing in a real diesel engine. The gas used to fill the chamber is usually Nitrogen, since it has similar properties to the air and prevents undesirable combustions. However, the chamber can be also filled with Helium or Sulfur hexafluoride $\left(\mathrm{SF}_{6}\right)$ if higher density values are desired for the same pressure levels. 
The chamber pressure is controlled by two valves: an intake valve connected to the gas bottle for filling the chamber and a second valve which adjusts the pressure and extracts the gas if necessary.

The measuring principle of the momentum test rig is based on the measurement of the impact force of the spray on a piezo-electric pressure sensor and is only valid if the sensor area is bigger than the impact area of the spray. Under this assumption, the impact force is equivalent to the momentum flux at the orifice outlet considering that the pressure in the chamber is uniform, the gravity effects are negligible and the direction of the fuel deflected is perpendicular to the sensor ([31][32]).

The right position of the injector with respect to the sensor can be checked through the window located on the side. This optical access is also useful for detecting any possible anomaly in the injection process, such as an orifice partially blocked.

\section{DESCRIPTION OF THE CFD APPROACH.}

\subsection{Cavitation modelling.}

There are three main approaches for modelling cavitating flows [13]: one-fluid models, two-phase flow models and interface tracking-capturing models. One-fluid models (also known as continuum flow models or even homogeneous mixture models) consider the cavitating flow as a homogeneous mixture of liquid and vapour, avoiding the resolution of the Navier Stokes equations for each phase. Besides the Navier Stokes equations, this approach needs and additional equation (equation of state) which usually relates the pressure with the density of the mixture. The second approach previously mentioned, the two-phase models, is based on the resolution of the Navier Stokes equations for the liquid and the vapour phase separately. The connection between both equations or both phases in this approach is done through momentum and mass transfer terms. The last group, the interface tracking- 
capturing models, assume the flow as two different immiscible fluids or phases with different and constant densities. The continuity, momentum and energy equations are solved for each phase and its interface is calculated independently.

The code used in the present work belongs to the first group, one-fluid models, and therefore consider the flow as a homogeneous mixture of liquid fuel and vapour fuel. It is implemented in OpenFOAM (B [28] under the names of cavitatingFoam, rasCavitatingFoam and lesCavitatingFoam according to the method used to model the turbulence (laminar, RANS and LES respectively). All these versions have been validated by Salvador et al. in calibrated orifices, one hole nozzles and multi-holes nozzles [8][13][20] [21] [33], showing a good agreement with experimental data in a wide range of pressure conditions and geometries.

The code assumes that the temperature of the fluid is constant and calculates the growth of the vapour phase due to cavitation with a barotropic equation of state (Eq. (1)). This equation relates the density and the pressure of the fluid through the compressibility, defined as the inverse of the speed of sound squared (Eq. (2)):

$$
\begin{aligned}
& \left(\frac{\partial \rho(t, P(\vec{x}, t))}{\partial P}\right)_{t}=\Psi \\
& \Psi=\frac{1}{c^{2}}
\end{aligned}
$$

The amount of vapour phase in each cell of the domain is represented by the variable $\gamma$, which can be calculated as a function of the mixture density, the liquid density at saturation and the vapour density at saturation (Eq. (3)). 


$$
\gamma=\max \left(\min \left(\frac{\rho-\rho_{l, s a t}}{\rho_{v, s a t}-\rho_{l, s a t}}, 1\right), 0\right)
$$

As can be seen from Eq. (3), $\gamma$ will be 0 if all the flow of the cell is in liquid phase and 1 if the cell is completely full of vapour.

With regard to the calculation of the compressibility of the fluid, a linear model is used (Eq. (4)) due to its good convergence and stability instead of more accurate models available in OpenFOAM (Chung [34] and Stewart [35]).

$$
\Psi=\gamma \Psi_{v}+(1-\gamma) \Psi_{l}
$$

Considering that there is only liquid or vapour and the speed of sound is constant, Eq. (1) can be written as follows:

$$
\begin{aligned}
& \rho_{v}=\Psi_{v} P \\
& \rho_{l}=\rho_{l}^{0}+\Psi_{l} P
\end{aligned}
$$

Finally, similar linear models are used as well for the calculation of the density and viscosity of the fluid:

$$
\begin{aligned}
& \rho=(1-\gamma) \rho_{l}^{0}+\Psi P \\
& \mu=\gamma \mu_{v}+(1-\gamma) \mu_{l}
\end{aligned}
$$

The methodology followed by the code begins with the resolution of the continuity equation (Eq. (9)) in order to obtain a density value from the initial velocity conditions. 


$$
\frac{\partial \rho}{\partial t}+\nabla \cdot(\rho \vec{u})=0
$$

Each term of the equation has been individually discretized, choosing the most suitable scheme in terms of accuracy and stability according to the particularities of the application. For the simulation of diesel injector nozzles, the authors determined in previous studies [13] that the divergence term $\nabla \cdot(\rho \vec{u})$ must be discretized in the space with a Gauss upwind scheme. The authors also concluded that an implicit discretisation in time must be used for the density in the divergence term whereas an Euler scheme was the most appropriate option for the partial derivative over time.

From the provisional density value obtained from Eq.(1), preliminary values of void fraction $(\gamma)$ and compressibility ( $\Psi)$ are computed using Eqs. (3) and (4). These values are introduced in the momentum conservation equation (Eq. (10)) in order to obtain the velocity from the initial pressure conditions. For this equation, a Gauss upwind scheme was used for the divergence terms and an Euler scheme was chosen for the partial derivatives over time.

$$
\frac{\partial(\rho \vec{u})}{\partial t}+\nabla \cdot(\rho \vec{u} \cdot \vec{u})=-\nabla P+\nabla \cdot\left(\mu\left(\nabla \vec{u}+\nabla \vec{u}^{T}\right)\right)
$$

Then, the pressure is calculated with an iterative PISO algorithm and the velocity is corrected through the continuity equation (Eq. (9)) turn into the next pressure equation through the barotropic equation of state (Eq. (7)):

$$
\frac{\partial(\Psi P)}{\partial t}-\left(\rho_{l}^{0}+\left(\Psi_{l}-\Psi_{v}\right) P_{v a p}\right) \frac{\partial}{\partial t}-P_{v a p} \frac{\partial \Psi}{\partial t}+\nabla \cdot(\rho \vec{u})=0
$$

Once reached the continuity condition, the density, void fraction and compressibility are updated with Eqs. (7), (4) and (3), and introduced again in the momentum equation until convergence. 


\subsection{Turbulence modelling}

As it was mentioned in the previous section, the code can be run in three different versions, depending on the method used to model the turbulence. The first and simplest version of the code is the laminar version, where the turbulence effects are not considered. This option is not suitable for the simulations run in the present study, since the Reynolds numbers reach up to 24,000 at high injection pressure. The second and third versions of the code compute the turbulence using RANS (Reynolds-averaged Navier-Stokes) and LES (Large Eddy Simulation) methods respectively.

For the simulations shown in the present paper, the turbulence has been modelled using a RANS method, which splits the solution into an averaged component and a fluctuating component. The submodel chosen was the RNG k- $\varepsilon$ model [36] with the Boussinesq assumption for modelling the turbulent viscosity $\left(\mu_{\mathrm{t}}\right)$ :

$$
\begin{aligned}
& -\overline{u_{i}^{\prime} u_{j}^{\prime}}=\mu_{t}\left(\frac{\partial \overline{u_{i}}}{\partial x_{j}}+\frac{\partial \overline{u_{j}}}{\partial x_{i}}-\frac{2}{3} \frac{\partial \overline{u_{k}}}{\partial x_{k}} \delta_{i j}\right)-\frac{2}{3} k \rho \delta_{i j} \\
& \mu_{t}=\rho C_{\mu} \frac{k^{2}}{\varepsilon}
\end{aligned}
$$

The RNG k- $\varepsilon$ model adds two new transport equations to the model: one for the turbulent kinetic energy $(k)$ and another one for the turbulent energy dissipation $(\varepsilon)$.

$$
\begin{aligned}
& \frac{\partial \rho k}{\partial t}+\nabla \cdot(\rho k \overline{\vec{u}})=\nabla \cdot\left[\left(\mu+\mu_{t} \alpha_{k}\right) \nabla k\right]+p_{k}-\rho \varepsilon \\
& \frac{\partial \rho \varepsilon}{\partial t}+\nabla \cdot(\rho \varepsilon \overline{\vec{u}})=\nabla \cdot\left[\left(\mu+\mu_{t} \alpha_{\varepsilon}\right) \nabla \varepsilon\right]+C_{\varepsilon 1} \frac{\varepsilon}{k} p_{k}-C_{\varepsilon 2}^{o} \rho \frac{\varepsilon^{2}}{k}
\end{aligned}
$$

with:

$$
C_{\varepsilon 2}^{o}=C_{\varepsilon 2}+\frac{C_{\mu} \eta^{3}\left(1-\frac{\eta}{\eta_{0}}\right)}{1+\beta \eta^{3}}
$$


The variables $p_{k}, \eta, S$ represent the production of turbulent kinetic energy, expansion parameter and the mean strain modulus respectively, and can be calculated as:

$p_{k}=\mu_{t} S^{2}$

$\eta=\frac{S k}{\varepsilon}$

$S=\sqrt{2 S_{i j} S_{i j}}$

The $C_{\varepsilon 1}, C_{\varepsilon 2}, \alpha_{k}, \alpha_{\varepsilon}, C_{\mu}, \eta_{0}$ and $\beta$ parameters are constants, whose values are:

$$
\begin{aligned}
& C_{\varepsilon 1}=1.42 \\
& C_{\varepsilon 2}=1.68 \\
& \alpha_{k}=1.39 \\
& \alpha_{\varepsilon}=1.39 \\
& C_{\mu}=0.0845 \\
& \eta_{0}=4.38 \\
& \beta=0.012
\end{aligned}
$$

\section{NOZZLES GEOMETRY AND CALCULATION SET-UP.}

\subsection{Nozzles geometry}

The geometrical features of the nozzles considered in this study are shown in Table 1. They are nozzles with six orifices. Two of them correspond to the geometries of the real nozzles that have been experimentally tested in the present work (CA60-r5 and CA155-r30). These nozzles have also been simulated and the numerical results have been contrasted against the experimental ones for code validation purposes. Moreover, an important set of additional nozzles comprises the basis for a computational study that has made it possible to analyse in an extensive manner the importance of 
the inclination and rounding axis on the internal flow in injection nozzles.

As it can be seen in the table, three values of orifice inclination angle and three values of rounding radius at their inlet have been considered. The combination of all possibilities leads to a total of nine nozzles. As described by the table, all the nozzle orifices are cylindrical, with a diameter of 170 micrometers. Considering that the nozzles with cylindrical orifices are prone to cavitate ([6]), this will make it possible to compare the geometries not only in terms of mass flow rate or momentum flux (directly related to the injection velocity) but also in terms of the critical conditions under which cavitation appears and its development. Given that the nozzles are symmetrical, only one sector including a single orifice is examined in the simulations. Figure 1 shows the geometry used for the simulations, where the geometrical parameters that have been evaluated can be observed: orifice inclination angle and rounding radius at the orifice inlet.

\subsection{Calculation setup.}

As it was previously mentioned, the calculations have been done for only one of the six orifices of each nozzle since they are completely symmetrical. This simplification can be applied as long as a symmetry boundary condition is set on both sides of the simulated geometry and represents an important saving of time due to the great number of simulations run in the present study: 189 cases resulting from the nine nozzles simulated at full needle lift, keeping the injection pressure at 1600 bar and varying the backpressure from 30 up to 500 bar in 21 different values.

All these simulations have been run using both the resources of the Spanish National Grid [37] (which is part of the European Grid Initiative [38]) and the supercomputer Tirant at the University of Valencia (Spain) [39]. In particular, 42,000 CPU hours were used from the Spanish National Grid Initiative 15,000 CPU hours from Tirant supercomputer (corresponding to those cases simulated at high backpressures). 
The meshing process has been performed following the conclusions obtained from previous sensitivity studies in similar nozzles ([13],[24]). These studies established that the cell size in the orifice must grow from $1.15 \mu \mathrm{m}$ in the vicinity of the wall up to $7 \mu \mathrm{m}$ in the centre of the orifice. For the rest of the domain (upstream of the orifice) a cell size of $22.5 \mu \mathrm{m}$ is enough to ensure the coherence and accuracy of all the flow variables calculated (velocity, pressure, density, turbulent kinetic energy, energy dissipation, etc.). Following these recommendations, the resulting meshes have around 240,000 hexahedral cells.

Regarding the boundary conditions applied to these meshes, the flow has been simulated setting two different kinds of pressure boundary conditions at the inlet section of the nozzle and at the exit of the nozzle orifice (see Figure 1). The injection pressure has been set as a fixed and uniform value whereas the backpressure has been set with a mean value boundary condition. This special boundary condition available in OpenFOAM, keeps the mean value of the desired pressure but allows at the same time the presence of flow at the nozzle outlet with higher or lower pressure values due to the presence of cavitating flow.

A representative injection pressure of real engine running conditions has been considered: $160 \mathrm{MPa}$. In order to characterize and compare all nozzles in different Reynolds regimes and in both cavitating and non-cavitating conditions, 21 different backpressure values have been simulated. All the pressure conditions simulated can be seen in Table 2. The backpressures used in the experimental facilities for the characterization of two of the nine nozzles are also provided in the table. In this case, the maximum value is limited due to mechanical constraints. The wide range of pressure conditions simulated have allowed the characterization of the nozzles in a broad range of Reynolds numbers and turbulence levels and the detection of the critical cavitations conditions.

Once described the geometry, the meshing process and the boundary conditions applied to the 
simulations only the specification of the fluid properties is missing to complete the description of the case setup. In this sense, the code requires the introduction of the density, viscosity and compressibility of the liquid and vapour phases of the fuel. The density and viscosity of the liquid phase have been taken from a commercial fuel (Repsol CEC RF-06-99) at $25^{\circ} \mathrm{C}$, whereas the compressibility value has been obtained from experimental tests where the speed of sound was measured [40]. As far as the vapour properties are concerned, they were obtained from Kärrholm et al. [41].

\section{EXPERIMENTAL RESULTS OF MASS FLOW AND MOMENTUM FLUX: MODEL} VALIDATION.

\subsection{Experimental results over nozzles CA155_r30 and CA60_r5.}

Figure 3 shows the results of mass flow rate and momentum flux for the two available nozzles, together with the effective injection velocity. This last parameter is obtained through the combination of the mass flow rate and momentum flux measurements, as shown in Eq. (20):

$$
u_{e f f}=\frac{\dot{M}_{f}}{m_{f}}
$$

As shown in Table 2, the results correspond to an injection pressure of $160 \mathrm{MPa}$ and eight different values of discharge pressure ranging between 3 and $17.5 \mathrm{MPa}$. This last value is close to the mechanical limits of the injection rate meter and the momentum flux test rig (Fig. 2), thus being the reason why the nozzles have not been characterized at higher backpressures. Each point represented corresponds to a discharge pressure, and the values of mass flow, momentum flux and velocity are obtained as mean values at maximum needle lift in stationary conditions. In order to obtain these parameters, the injector has been energized for $3 \mathrm{~ms}$, which is enough to ensure that those stationary 
conditions are attained ([20]). The momentum flux, measured as the impingement force of the jet on a pressure sensor calibrated to measure force, is one of the most important parameters on which the spray behaviour depends ([42][43]). In addition, this measurement combined with the mass flow results provides the effective injection velocity, as established by Eq. (20). In the same Figure, the computational results for these geometries are also represented and will be commented in the next section, which deals with the model validation.

As far as the experimental results shown in the Figure are concerned, the following observations can be made:

- Mass flow rate: The experimental points show a mass flow rate collapse, or non-dependence of the mass flow on the square root of the pressure drop for both nozzles. This phenomenon is well-known and is distinctive of cavitating nozzles, indicating that cavitation is taking place inside the nozzles holes ([13][20][44][45]) for all the backpressures tested. In comparative terms, the nozzle with the highest inclination (CA155_r30) shows a lower mass flow rate than the nozzle with the lowest inclination (CA60_r5). A priori, the lower deflection suffered by the streamlines at the orifice inlet (lower losses) in the latter, as will be investigated in another Section, could help to justify this behaviour. However, the differences in mass flow seen in this region strongly depend on the different critical conditions of cavitation inception (backpressure for which the cavitation appears inside the orifice) for both nozzles and their behaviour in non-cavitating conditions. In order to determine the critical conditions of cavitation inception, as well as to compare the behaviour of both nozzles in those conditions, we will rely on the results of the simulations, for which there is no limitation in the discharge pressure. Thus, the discharge pressure can be increased as far as needed in order to seek for the change in regime: from cavitating to non-cavitating. This result will be commented in the next section about code validation. For now, the focus is given to the purely experimental 
results represented in the Figure, both for mass flow, momentum flux and effective injection velocity.

- Momentum flux: Despite having found that the flow inside the nozzle orifices is cavitating through the previously commented mass flow measurements, the momentum flux measurements do not show collapse, i.e. increases linearly with the square root of the pressure drop. This result had already been noted widely both experimentally and numerically ([7][8][13][20][21]). Comparatively, the nozzle with the lowest inclination angle (CA60_r5) exhibits a higher momentum flux at the orifice outlet than the nozzle with the highest inclination angle (CA155_r30). In this case, although the differences are small, the better predisposition of the nozzle CA60_r5 for the flow entrance would justify again the observed behaviour. It must be noted that, even though the lower inclination angle facilitates the fuel entrance to the orifice, the higher rounding radius of the nozzle with a higher inclination would act in the sense of reducing the differences, since a higher rounding radius helps to minimize the flow detachment at the entrance. As a consequence, the differences are reduced, but a higher influence of the inclination as compared to the radius is noticed, since the nozzle CA60_r5 shows values slightly higher than those of the nozzle CA155_r30.

- Effective injection velocity: It is a parameter derived from the previous measurements as stated by Eq. (20). In both cases, the fact that the mass flow remains collapsed as a consequence of cavitation, and that the momentum flux increases with the square root of the pressure drop, results in an increase of the effective injection velocity. This increase is higher than the one that could be expected by the mere fact of increasing the pressure drop, and is a direct consequence of the existence of cavitation inside the orifice, which directly results in a viscosity reduction near the wall region (where the vapour is located) and a turbulent velocity profile flattening that originates an increase in the effective injection velocity ([13]). For the 
aforementioned reasons, the differences are small, favoring the nozzle CA155_r30 in this case. Indeed, the increase in effective injection velocity with cavitation gets more important the higher the cavitation intensity inside the orifice is. In a following Section, when the results of the simulations are analysed and the critical cavitation conditions are determined, it will be seen that the nozzle CA155_r30 starts cavitating for higher backpressure values, which means that is more prone to cavitate. As a consequence, for the experimentally tested backpressures the cavitation intensity is higher for this nozzle, and thus their impact on the effective injection velocity (resulting in its increase) is higher than for the CA60_r5.

\subsection{Code validation.}

In Figure 3, together with the experimental data previously analysed, the results from the numerical simulations performed by using geometries that reproduce the tested nozzles are shown. In this case, as stated previously, since there is no limitation in the backpressure values, the comparison may be extended to low values of the square root of the pressure drop, i.e. low values of Reynolds number. This fact also makes it possible to determine the cavitation critical conditions, namely the value of backpressure that produces the cavitation inception for the injection pressure considered (invariant and equal to $160 \mathrm{MPa}$ ), from which its reduction results in the mass flow collapse previously commented.

From the comparison among the experimental and computational results, it can be seen that the calculation code is able to predict the experimental results with a reliability of about $4 \%$ or $5 \%$. The same level of accuracy had been found in other validations of the code reported in previous studies ([20][33]). It is also interesting to note that these differences remain for the results of both nozzles, 
which provides the results presented in the following Section a higher reliability, such that when the differences among nozzles are qualitatively assessed, those differences are representative of the ones existing in reality.

The results for the backpressure conditions experimentally tested have already been discussed. As far as the results in the non-cavitating zone (where the mass flow recovers the linear dependence with the square root of the pressure drop) are concerned, it can be highlighted that the differences found in terms of mass flow, momentum flux and effective velocity are very small and, in general terms, in favour of the CA60_r5 nozzle. As it has been commented previously, the combination of the inclination and rounding radius values between nozzles makes the differences amongst them significantly reduced instead of being enhanced. Where there is indeed an important difference among nozzles is in the critical cavitation conditions. These conditions can be characterized either through the backpressure level for which the flow starts being collapsed, or through the critical cavitation number. There are several definitions for the cavitation number ([6] [9][46] [47]). One of them is the parameter $K$, defined as a function of injection pressure $\left(P_{i n j}\right)$, the backpressure $\left(P_{b a c k}\right)$, and the vaporization pressure $\left(P_{\text {vap }}\right)$ as Eq. (21) states. Given that the fuel vaporization pressure is much lower than the injection pressure, it is usual to disregard the term $\left(P_{v a p}\right)$ in the numerator:

$$
K=\frac{P_{i n j}-P_{\text {vap }}}{P_{\text {inj }}-P_{\text {back }}}
$$

The way this parameter is defined, as the backpressure is reduced for a given injection pressure, the denominator grows larger and the numerator remains constant. This means that, the greater the pressure difference the nozzle is submitted to, the lower the value of $K$. The value of $K$ related to the critical cavitation conditions is named as the critical cavitation number, $K_{\text {crit }}$.

The critical cavitation conditions, both in terms of discharge pressure and $K_{\text {crit }}$ values for both nozzles, are $28.57 \mathrm{MPa}$ and $K_{\text {crit }}=1.218$ for the nozzle CA60_r5 and $36.12 \mathrm{MPa}$ and $K_{\text {crit }}=1.29$ for the nozzle 
CA155_r30. These values highlight the higher proneness of the CA155-r30 nozzle to cavitate.

The geometrical configuration of the previously studied nozzles does not make it possible to isolate the influence of each of the considered geometrical parameters (namely inclination and rounding radius) on the flow. In order to somehow quantify the relative importance of each of them, thus establishing the maximum differences that can be found in practice among nozzles due to different inclination values or hydro-erosion levels, a purely computational study is performed next. In this study, as it was reflected in Table 1, three levels of inclination (including the two values experimentally examined) and three values of rounding radius (including the two ones tested) are combined.

\section{COMPUTATIONAL RESULTS AND ANALYSIS.}

In order to analyze the results of the comparison among different nozzles, and in order to make them independent from the nozzle diameter, some parameters that make it possible to characterize the flow behaviour are defined in this section.

\subsection{Definition of dimensionless parameters characterizing the inner nozzle flow.}

Non-dimensional flow coefficients are useful to analyze the behaviour of the flow in terms of mass flow (pressure losses), effective velocity and effective injection section of the orifice.

The most important dimensionless parameter is the discharge coefficient, $C_{d}$, which is defined as the mass flow divided by the maximum theoretical mass flow related to the maximum velocity of the flow given by the Bernoulli's equation:

$C_{d}=\frac{m_{f}}{\rho_{l} A_{o} u_{t h}}=\frac{m_{f}}{A_{o} \sqrt{2 \rho_{l} \Delta P}}$

where $\dot{m}_{f}$ is the mass flow, $\Delta \mathrm{P}$ is the difference between the injection pressure $\left(P_{i n j}\right)$ and the 
backpressure $\left(P_{\text {back }}\right), \Delta P=P_{i n j}-P_{b a c k}, A_{o}$ is the geometrical area of the outlet of the orifice and $\rho_{l}$ is the liquid fuel density.

The second non-dimensional flow parameter is the velocity coefficient, $C_{v}$, which relates the effective velocity to the maximum theoretical Bernoulli velocity, $u_{t h}$ :

$$
\begin{aligned}
& u_{t h}=\sqrt{\frac{2\left(P_{\text {inj }}-P_{\text {back }}\right)}{\rho}} \\
& C_{v}=\frac{u_{e f f}}{u_{t h}}
\end{aligned}
$$

The effective velocity can be calculated by dividing the momentum flux by the mass flow as was established by equation (20)

The third flow coefficient, $C_{a}$, is used to evaluate the reduction of the effective area with regard to the geometric one due to the presence of vapour bubbles into the flow that reach the orifice outlet

$$
C_{a}=\frac{A_{e f f}}{A_{o}}
$$

Flow parameters are related to each other by means of equation (26):

$$
C_{d}=C_{v} C_{a}
$$

\subsection{Analysis of results.}

\subsubsection{Comparison in terms of mass flow, momentum flux, effective velocity and non dimensional parameters.}

Fig. 4 shows the results of the CA155 nozzle for the three different values of rounding radius where, for the CA155_r30 nozzle, the results coincide with those presented previously. The results are shown in terms of mass flow, momentum flux, effective velocity and the three non-dimensional coefficients 
obtained according to the definitions made in Section 6.1. The mass flow, momentum flux and effective velocity results show the same behaviour as the one described in Sections 5.1 and 5.2.

When the three cases are compared, the strong influence of the rounding radius at the orifice inlet is highly noticed: the lower the radius, the lower the values obtained for the three variables, drawing differences among them up to a $10 \%$ in terms of mass, momentum flux and effective velocity. This is explained due to the higher pressure losses the flow suffers the lower the rounding radius gets, since this situation leads to a more important flow detachment. These higher losses are translated into a lower discharge coefficient, as the lower part of the figure shows. On the other hand, the discharge coefficient shows a peculiar behaviour due to the mass flow collapse. From the highest value reached in the non-cavitating zone, there is an abrupt fall when the cavitation inception conditions are reached. These critical conditions are shown in Table 3 in terms of discharge pressure and $K_{\text {crit }}$ where, in addition to the three CA155 nozzle cases, the other simulated nozzles whose results are commented along this section also appear. From these results it can be derived that the proneness of a nozzle to cavitate is enhanced the lower its rounding radius. The reason has to do with what was previously commented: the more important detachment of the flow when the radius is reduced induces a higher flow recirculation and a more drastic reduction of the pressure in the critical zone.

With regard to the area coefficient, it shows values around the unity in non-cavitating conditions for the three cases, whereas it experiences a strong diminution once the cavitation appears. The opposite is true for the velocity coefficient, which experiences an important increase from the critical conditions that grows larger as the cavitation is intensified.

The same kind of information is represented for the nozzles CA145_r5, CA145_r13 and CA145_r30 (Fig. 5) and CA60_r5, CA60_r13 and CA60_r30 (Fig. 6). In qualitative terms, the behaviour observed when comparing the three hydro-erosion levels for a certain inclination angle is the same that was 
previously described for the CA155 series: the lower the rounding radius, the higher the losses and the more prone to cavitate the nozzle is (Table 3). In quantitative terms, if the parameters evaluated for the three inclination levels are compared, it can be appreciated that in general terms the CA60 series show the higher values of mass flow, momentum flux and effective velocity (and their corresponding non-dimensional parameters), followed by the CA145 series and finally the CA155 series. However, depending on the value of the rounding radius, the differences among nozzles are reduced. A clear example of this fact is the experimental comparison performed in Section 5.1 among the CA155_r30 and CA60_r5 nozzles, where it had already been noted that the differences are reduced due to the opposed effects of the inclination and the rounding radius. This can be clearly appreciated in Figures 7 to 10, where the mass flow, discharge coefficient, momentum flux and effective injection velocity are respectively represented for all the nozzles. As it has already been mentioned, the highest values are registered for the nozzles of the CA60 series, followed by the CA145 and the CA155. If the extreme cases are compared (CA60_r30, more favourable from the point of view of the flow; and CA155_r5, less favourable from that same point of view) and the differences are computed for a backpressure value of $9 \mathrm{MPa}\left(\sqrt{\Delta P}=12.2 M P a^{0.5}\right)$, which could be a characteristic engine operating point, differences of $16 \%$ in terms of discharge capacity (mass flow) and up to an $8 \%$ in terms of effective velocity are observed. However, out of these extreme values, at intermediate levels, situations can be found in which a nozzle with higher inclination but a larger radius show better performance than a nozzle with lower angle and small radius. That is the case of the CA155_r30 and CA145_r5 nozzles, for instance.

Fig. 11, in its upper side, shows a map of discharge coefficient as a function of the orifice angle and the rounding radius for the reference case previously considered $\left(P_{i n j}=160 \mathrm{MPa}, P_{b a c k}=9 \mathrm{MPa}\right)$. The discharge coefficient has been chosen since it includes all kinds of losses. It is clearly noticed that the highest value of this coefficient is obtained for the lower inclinations and higher values of radius. The 
lowest value would then be obtained for the higher inclinations and lower entrance radius.

As it has been said, the critical cavitation conditions are strongly dependent on both geometrical parameters. In order to condense the information provided in Table 3 about the critical cavitation number, the variation of $K_{\text {crit }}$ as a function of the inclination and the rounding radius has been represented in the lower part of Fig. 11. The map clearly shows how the less cavitating configurations (lower $K_{c r i t}$ ) would be found for the nozzles with lower inclinations and higher entrance radius.

\subsubsection{Cavitation morphology}

In Figs. 12 and 13 the vapour field in a mid-section of the orifice is represented, associated to the mass flow results previously analysed for nozzles CA155_r13 and CA60_r13, respectively. An interesting result that had already been noted in the past ([13]) is the fact that the first appearance of vapour (incipient cavitation) takes place before the mass flow collapse. It can be said that the entity of the generated vapour is not enough to produce that collapse. From that value of backpressure, as the cavitation intensity grows as the backpressure is reduced, extending the vapour from the upper corner of the entrance, where it is originated, towards the orifice outlet. The most important difference between the two nozzles is the region in which cavitation appears. In the case of the CA155 nozzle, it is originated in the upper radius of the orifice inlet, whereas in the case of the CA60 nozzles the opposite takes place and cavitation appears in the lower radius at the orifice inlet. This difference also generates a variance in the way the vapour is extended as the backpressure is reduced. In the case of the CA155 nozzle, the vapour extends along the wall and stuck to it, whereas in the CA60 nozzle there is a tendency of the generated vapour to extend towards the centre of the orifice. The reason why the point of origin of cavitation is different in both nozzles can be explained from the fluid streamlines representation shown in Fig. 14 for both cases. As it can be clearly seen, in the case of the CA155 nozzle the orifice inclination favors a detachment of the flow that mainly comes from the 
upper part of the nozzle, channeled between the needle wall and the nozzle seat. That detachment takes place in the upper corner of the inlet. It is in this zone where there appear some local vortices with flow acceleration that imply a pressure reduction down to values below the vapour pressure. In the case of the CA60 nozzle, the configuration makes the detachment take place in the lower corner of the inlet and with less importance on the upper part, or at least not enough to originate cavitation in that region.

\section{CONCLUSIONS}

The results obtained along the present study make it possible to establish the following conclusions:

- From the experimental study:

- The mass flow results show a collapse for all the studied conditions (several backpressures for an injection pressure of $160 \mathrm{MPa}$ ), indicating cavitation. The nozzle with a low inclination angle (CA60_r5) exhibits a higher mass flow than the one with the high inclination angle (CA155_r30), justified by the lower deflection suffered by the streamlines in the former.

- With regard to the momentum flux, where there is no collapse, CA60_r5 nozzle also shows higher values than the CA155_r30 for all the tested conditions, although the differences are small. This is due to the opposed effects of the inclination and the rounding radius: whereas a lower inclination favours the flow entrance, a higher radius minimises the flow detachment at the inlet. A higher influence of the inclination than that of the rounding radius is thus noted.

- As far as the effective injection velocity is concerned, the cavitation phenomenon 
tends to increase its values. Therefore, the CA155_r30 nozzle, which exhibited a higher cavitation intensity as confirmed by the computational results, shows higher velocities than the CA60_r5 nozzle. In any case, the differences are small since this parameter is derived from the former two.

- From the code validation:

- The comparison against the experimental results shows a reliability level of around 4 or $5 \%$, consistent among nozzles, which makes it possible to quantitatively assess the differences among nozzles along the whole study.

o The simulations have been extended to the non-cavitating conditions, making it possible to determine the cavitation critical conditions. The differences in terms of mass flow, momentum flux and effective velocity among nozzles in this region are small. However, important differences are noticed with regard to the critical cavitation conditions, highlighting the higher proneness of the CA155_r30 nozzle to cavitate.

- From the computational results:

- Simulations have been performed combining three levels of inclination angle and three levels of rounding radius, leading to 9 different nozzles that have been evaluated. Comparisons have been made not only in terms of mass flow, momentum flux and effective velocity, but also in terms of the non-dimensional parameters that characterize the flow and its losses, namely discharge coefficient, area coefficient and velocity coefficient.

- The general behaviour of the variables observed from the experimental tests and the code validation are confirmed by the simulations. 
- A strong influence of the rounding radius has been noted, leading to differences up to $10 \%$ in terms of mass flow, momentum flux and effective velocity among the extreme cases. This can be justified by the flow detachment, enhanced by low values of the radius. The analysis of the critical cavitation conditions also make it possible to state that a nozzle is more prone to cavitate the lower its rounding radius.

- With regard to the influence of the inclination angle, the CA60 series show higher values of mass flow, momentum flux and effective velocity than the CA145 and the CA155 series in general, confirming the trend that had already been observed experimentally.

When assessing the combined influence of the inclination angle and the rounding radius, that lead to opposed effects on the flow, if the extreme cases (CA60_r30 nozzle versus CA155_r5) are compared, differences up to a $16 \%$ in terms of mass flow and $8 \%$ in terms of momentum flux are observed. However, due to the opposed effects of these variables, the differences among series are reduced for intermediate cases, even leading to situations in which a nozzle with a higher inclination but a large radius exhibit a better performance than a nozzle with a lower angle and small radius.

- As it was expected, the cases with lower inclination angles and higher rounding radius lead to the higher discharge coefficient values and the less cavitating configurations.

- As far as the cavitation morphology is concerned, it is important to note that in the case of a high inclination angle, cavitation is originated at the upper corner of the orifice entrance, and its evolution takes place along the wall when the cavitation intensity is enhanced. However, in the case of a low inclination angle, cavitation starts at the lower corner of the orifice entrance, and it tends to displace towards the orifice 
centre when the cavitation intensity grows larger.

\section{ACKNOWLEDGEMENTS}

This work was partly sponsored by "Ministerio de Economía y Competitividad" in the frame of the project "Comprensión de la influencia de combustibles no convencionales en el proceso de inyección y combustión tipo diesel”, Reference TRA2012-36932. The equipment used in this work has been partially supported by FEDER project funds "Dotación de infraestructuras científico técnicas para el Centro Integral de Mejora Energética y Medioambiental de Sistemas de Transporte (CiMeT), (FEDER-ICTS-2012-06)", in the frame of the operation program of unique scientific and technical infrastructure of the Ministry of Science and Innovation of Spain. This support is gratefully acknowledged by the authors.

The authors would like to thank the computer resources, technical expertise and assistance provided by the Universidad de Valencia in the use of the supercomputer "Tirant".

\section{REFERENCES}

[1] Bermúdez, V., Payri, R., Salvador, F.J., Study of the influence of nozzle seat type on injection rate and spray behaviour, Proceedings of the institution of mechanical engineers Part-D, Journal of automobile engineering, 219 issue D5 (2005), pp. 677-689.

[2] Payri, R., Tormos, B., Salvador, F.J., Spray droplet velocity characterization for convergent nozzles with three different diameters, FUEL, 87 (2007), pp. 3176-3182. 
[3] Payri, R., Salvador, F.J., Gimeno, J., Soare, V., Determination of Diesel sprays characteristics in real engine in cylinder air density and pressure conditions, Journal of Mechanical Science and Technology, 19 (2005), pp. 2040-2052.

[4] Andriotis, A., Gavaises, M., Influence of vortex flow and cavitation on near-nozzle diesel spray dispersion angle, Atomization and sprays, 19 (3) (2009), pp. 247-261.

[5] Li, X., Chen, C., Yu, Y., Gao, G., Numerical investigation on effects of nozzle's geometric parameters on the flow and the cavitation characteristics within injector's nozzle for a highpressure common-rail DI diesel engine, Energy Conversion and Management, 89 (2015), pp. 843-864.

[6] Payri, F., Bermúdez, V., Payri, R., Salvador, F.J. The influence of cavitation on the internal flow and the spray characteristics in diesel injection nozzles, Fuel, 83 (2004), pp. 419-431.

[7] Desantes, J.M., Payri, R., Salvador, F.J., De la Morena, J., Influence of cavitation phenomenon on primary break-up and spray behavior at stationary conditions, Fuel, 89 (2010), pp. 3033-3041.

[8] Salvador, F. J., Romero, J. -V., Rosello, M. -D., Martínez-López, J., Validation of a code for modeling cavitation phenomena in diesel injector nozzles. Mathematical and Computer modelling, 52 (2010), pp. 1123-1132.

[9] Schmidt, D.P., Corradini, M.L., The internal Flow of Diesel Fuel Injector Nozzles: a Review, International Journal of Engine Research, 2 (1) (2001).

[10] Winklhofer, E., Kull, E., Kelz, E. Morozov, A. Comprehensive hydraulic and flow field documentation in model throttle experiments under cavitation conditions, ILASS Europe, 2001.

[11] Chaves, H. and Schuhbauer, L. Cavitation in an asymmetric transparent real size VCO nozzle, proceedings of Spray’06, 2006. 
[12] Gavaises, M., Andriotis, A., Papolias, D., NMitroglou, N., Theodorakakos, A., Characterization of string cavitation in large-scale diesel nozzles with tapered holes, Physics of Fluids, 21 (2009).

[13] Payri, F., Payri, R., Salvador, F.J., Martínez-López, J., A contribution to the understanding of cavitation effects in diesel injector nozzles through a combined experimental and computational investigation, Computer and Fluids, 58 (2012), pp. 88-101.

[14] Postrioti, L., Battistoni, M., Ungaro, C., Mariani, A., Analysis of diesel spray momentum flux spatial distribution, SAE International Journal of Engines, 4(1) (2011), pp. 720-736.

[15] Sangiah, D., Ganippa, L., Application of spray impingement technique for characterization of high pressure sprays from multi-hole diesel nozzles, International Journal of Thermal Sciences, 49(2) (2010), pp. 409-417.

[16] Alajbegovic, A., Meister, G., Greif, D., Basara. B., Three phase cavitating flows in high pressure swirl injectors, Experimental Thermal and Fluid Science, 26 (2002), pp. 677-681.

[17] Andriotis, A., Gavaises, M., Influence of vortex flow and cavitation on near-nozzle diesel spray dispersion angle, Atomization and Sprays, 19(3) (2009), pp. 247-261.

[18] Schmidt, D.P., Rutland, C., Corradini, M., A fully compressible two-dimensional model of high speed cavitating nozzles, Atomization and sprays, 9 (1999), pp. 255-276.

[19] Karrholm, F., Weller, H., Nordin, N., Modelling injector flow including cavitation effects for diesel applications, $5^{\text {th }}$ Joint ASME/JSME Fluids engineering Conference, 2007.

[20] Salvador, F.J., Hoyas, S., Novella, R., Martínez-López, J., Numerical simulation and extended validation of two-phase compressible flow in diesel injector nozzles, Proceedings of the institution of mechanical engineers part-D-Journal of automobile engineering, vol. 225, (2011), pp. 545-563. 
[21] Salvador, F.J., Martínez-López, J., Caballer, M., de Alfonso, C., Study of the influence of needle lift on the internal flow and cavitation phenomenon in diesel injector nozzles by CFD using RANS methods, Energy conversion and management, vol. 66 (2013), pp. 246-256.

[22] de la Morena, J., Neroorkar, K., Plazas, A. H., Peterson, R. C., Schmidt, D. P., Numerical analysis of the influence of diesel nozzle design on internal flow characteristics for 2-valve diesel engine application, Atomization and sprays, 23 (2) (2013), pp. 97-118.

[23] Molina, S., Salvador, F.J., Carreres, M. Jaramillo, D., A computational investigation on the influence of the use of elliptical orifices on the inner nozzle flow and cavitation development in diesel injector nozzles, Energy conversion and management, 79 (2014), pp. 114-127.

[24] Macián, V., Payri, R., Margot, X. Salvador, F.J., A CFD analysis of the influence of diesel nozzle geometry on the inception of cavitation, Atomization and sprays, 13 (2003), pp. 579604.

[25] Kampmann, S., Dittus, B., Mattes, P., Kirner, M., The influence of hydro-grinding at VCO nozzle on the mixture preparation in a DI diesel engine, SAE Paper 960867 (1996).

[26] http://www.transportation.anl.gov/engines/fuel_injection_sprays_dynamic_imaging.html

[27] Fiorentino, L., Laforgia, D., Bruni, G., Fiore, A., 3-D analysis of the flow through a multihole VCO nozzle for D.I. diesel engine, ATA April, 49(4) (1996).

[28] OpenFOAM is a registered trade mark of OpenCFD Limitedhttp://www.opencfd.co.uk/openfoam/index.html

[29] Marcic, M., Measuring method for Diesel Multihole Injection Nozzles, Sensors and actuators, 107 (2003), pp. 152-158. 
[30] Payri, R., Salvador, F.J., Gimeno, J., Bracho, G., A new methodology for correcting the signal cumulative phenomenon on injection rate measurements, Experimental techniques, 32(1) (2008).

[31] Payri, R., Ruiz, S., Salvador, F.J., Gimeno, J., On the dependence of spray momentum flux in spray penetration: Momentum flux packets penetration model, Journal of Mechanical Science and Technology, 21(7) (2007),pp 1100-1111.

[32] Salvador, F.J., Ruiz, S., Gimeno, J., De la Morena, J., Estimation of a suitable Schmidt number range in diesel sprays, International Journal of Thermal Sciences 50 (2011), pp.17901798

[33] Salvador, F.J., Martínez-López, J., Romero, J.-V., Roselló, M.-D., Computational study of the cavitation phenomenon and its interaction with the turbulence developed in diesel injector nozzles by Large Eddy Simulation (LES), Mathematical and Computer Modelling, 57 (2013), pp. 1656-1662.

[34] Chung, T.J., Computational Fluid Dynamics, second edition, Cambridge University Press (2010) ISBN 978-0-521-76969-3.

[35] Stewart, H.B., Wendroff, B., Two-phase flow: models and methods, J. Comput. Phys., 56 (1984), pp. 363-409.

[36] Yakhot, V., Orszag, S.A., Thangam, S., Gatski, T.B., Speziale, C.G., Development of turbulence models for shear flows by a double expansion technique, Phys. Fluids A 4 (1992), 1510.

[37] Spanish National Grid Initiative http://www.es-ngi.es/

[38] European Grid Initiative http://www.egi.eu 
[39] Tirant Node, http://www.bsc.es/annualreport/2009/wikiar.bsc.es/index.php5/Universidad_de_Valencia___Tirant_Node.html

[40] Payri, R., Salvador, F.J., Gimeno, J., Bracho, G., The effect of temperature and pressure on thermodynamic properties of diesel and biodiesel fuels, Fuel 90 (2011), 1172-1180.

[41] Peng Kärrholm, F., Weller, H., Nordin, N., Modelling injector flow including cavitation effects for Diesel Applications, Proceedings of FEDSM2007, 5th Joint ASME/JSME Fluids Engineering Conference, July 30- August 2, San Diego, California, USA.

[42] Desantes, J.M., Payri, R., García, J.M., Salvador, F.J., A contribution to the understanding of isothermal diesel spray dynamics, Fuel, 86 (2007), pp. 1093-110.

[43] Payri, R., Salvador, F.J., Gimeno, J., Gil, A., Development and validation of a theoretical model for Diesel spray penetration, Fuel, 85 (2006), pp. 910-917.

[44] Schmidt, D.P., Rutland, C.J., Corradini, M.L, A fully comprenssible two-dimensional model of high speed cavitating nozzles, Atomization and sprays, 9 (2009).

[45] López, J.J, Salvador, F.J., De la Garza, O.A. Arrègle, J., A comprehensive study on the effect of cavitation on injection velocity in diesel nozzles, Energy Conversion and Management, 64 (2012), pp. 415-423.

[46] Arcoumanis, C., Flora, H., Gavaises, M., Kampanis, N., Horrocks, R., Investigation of cavitation in a vertical multi-hole diesel injector. SAE Paper 1999-01-0524.

[47] Soteriou, C., Andrews, R., Smith, M., Further studies of cavitation and atomization in diesel injection. SAE Paper 1999-01-1486. 
Table 1: Nozzle's geometrical characteristics.

Table 2: Injection pressure and backpressures used in experiments and in simulations.

Table 3: Critical cavitation conditions in terms of backpressures needed for the inception of cavitation at maximum needle lift $(250 \mu \mathrm{m})$ and critical cavitation number $\left(K_{\text {crit }}\right)$

Figure 1: Sketch of the nozzle configuration including the geometrical definition of the parameters investigated.

Figure 2: Momentum test rig.

Figure 3: Comparison between experimental and numerical results for the nozzles CA60_r5 and CA155_r30.

Figure 4: Mass flow rate, momentum flux and effective velocity for the nozzle in the upper part, and non-dimensional flux parameters in the lower part for the Nozzles CA155 at $160 \mathrm{MPa}$ of injection pressure and different backpressures.

Figure 5: Mass flow rate, momentum flux and effective velocity for the nozzle in the upper part, and non-dimensional flux parameters in the lower part for the Nozzles CA145 at $160 \mathrm{MPa}$ of injection pressure and different backpressures.

Figure 6: Mass flow rate, momentum flux and effective velocity for the nozzle in the upper part, and non-dimensional flux parameters in the lower part for the Nozzles CA60 at $160 \mathrm{MPa}$ of injection pressure and different backpressures.

Figure 7: Comparison in terms of mass flow rate for all the nozzles.

Figure 8: Comparison in terms of discharge coefficient for all the nozzles.

Figure 9: Comparison in terms of momentum flux for all the nozzles.

Figure 10: Comparison in terms of effective velocity for all the nozzles.

Figure 11: Discharge coefficient as a function of orifice inclination and rounding radius for the injection condition $\mathrm{P}_{\mathrm{inj}}=160 \mathrm{MPa}$ and $\mathrm{P}_{\text {back }}=9 \mathrm{MPa}$ (upper part) and $\mathrm{K}_{\text {crit }}$ as a function of the half of the orifice angle $(\mathrm{CA} / 2)$ and the rounding radius.

Figure 12: Vapour field and mass flow for the nozzle CA155 for the injection pressure of $160 \mathrm{MPa}$ and different backpressures.

Figure 13: Vapour field and mass flow for the nozzle CA60 for the injection pressure of $160 \mathrm{MPa}$ and different backpressures.

Figure 14: Differences in streamlines between nozzles CA145_r13 and CA60_r13 


\begin{tabular}{|c|c|c|c|c|c|}
\hline 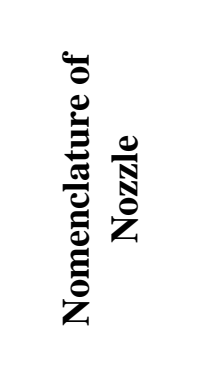 & 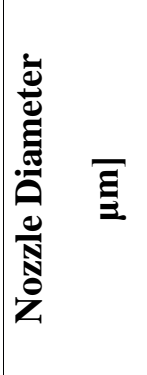 & 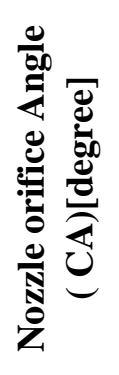 & 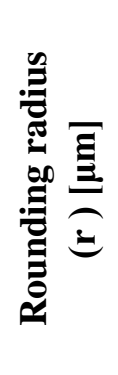 & 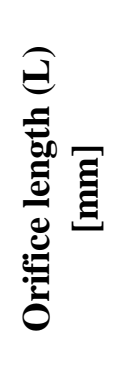 & 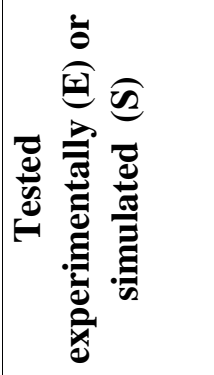 \\
\hline CA60_r5 & 170 & $60^{\circ}$ & 5 & 1 & E and $S$ \\
\hline CA60_r13 & 170 & $60^{\circ}$ & 13 & 1 & S \\
\hline CA60_r30 & 170 & $60^{\circ}$ & 30 & 1 & $S$ \\
\hline CA145_r5 & 170 & $145^{\circ}$ & 5 & 1 & $S$ \\
\hline CA145_r13 & 170 & $145^{\circ}$ & 13 & 1 & $S$ \\
\hline CA145_r30 & 170 & $145^{\circ}$ & 30 & 1 & $\mathrm{~S}$ \\
\hline CA155_r5 & 170 & $155^{\circ}$ & 5 & 1 & $\mathrm{~S}$ \\
\hline CA155_r13 & 170 & $155^{\circ}$ & 13 & 1 & $\mathrm{~S}$ \\
\hline CA155_r30 & 170 & $155^{\circ}$ & 30 & 1 & $E$ and $S$ \\
\hline
\end{tabular}

Table 1: Nozzle's geometrical characteristics.

\begin{tabular}{|c|c|c|}
\hline Injection Pressure [MPa] & \multicolumn{2}{|c|}{ Backpressure [MPa] } \\
\hline \multirow{2}{*}{160} & Experiments & $3,5,7,9,10,12.5,15$ and 17.5 \\
\cline { 2 - 3 } & \multirow{2}{*}{ Simulations } & $3,5,7,9,10,12.5,15,17.5,20,22.5,25,27.5,30$, \\
& & $32.5,35,37.5,40,42.5,45,47.5$ and 50 \\
\hline
\end{tabular}

Table 2: Injection pressure and backpressures used in experiments and in simulations. 


\begin{tabular}{|c|c|c|}
\hline 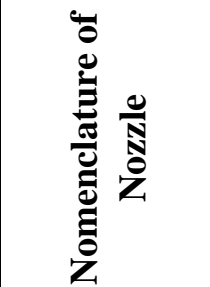 & 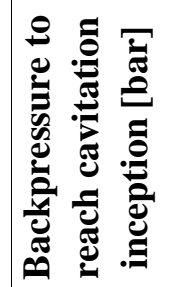 & 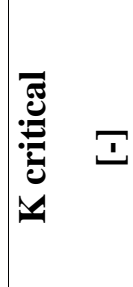 \\
\hline CA60_r5 & 285.80 & 1.218 \\
\hline CA60_r13 & 284.94 & 1.217 \\
\hline CA60_r30 & 260.76 & 1.195 \\
\hline CA145_r5 & 352.89 & 1.283 \\
\hline CA145_r13 & 337.33 & 1.267 \\
\hline CA145_r30 & 319.50 & 1.250 \\
\hline CA155_r5 & 374.89 & 1.306 \\
\hline CA155_r13 & 373.70 & 1.305 \\
\hline CA155 r30 & 361.23 & 1.292 \\
\hline
\end{tabular}

Table 3: Critical cavitation conditions in terms of backpressures needed for the inception of cavitation at maximum needle lift $(250 \mu \mathrm{m})$ and critical cavitation number $\left(K_{\text {crit }}\right)$ 


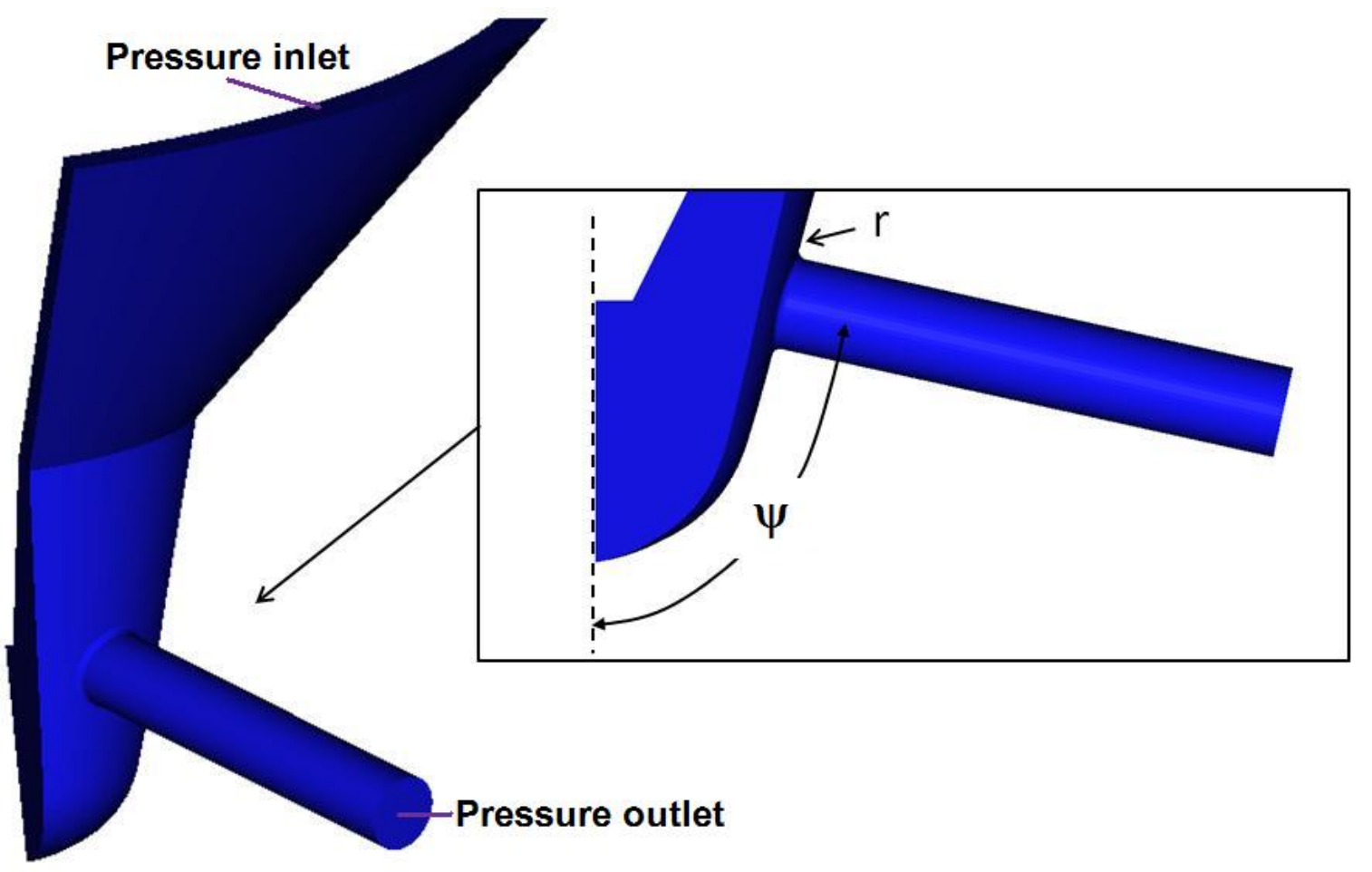

Figure 1: Sketch of the nozzle configuration including the geometrical definition of the parameters investigated.

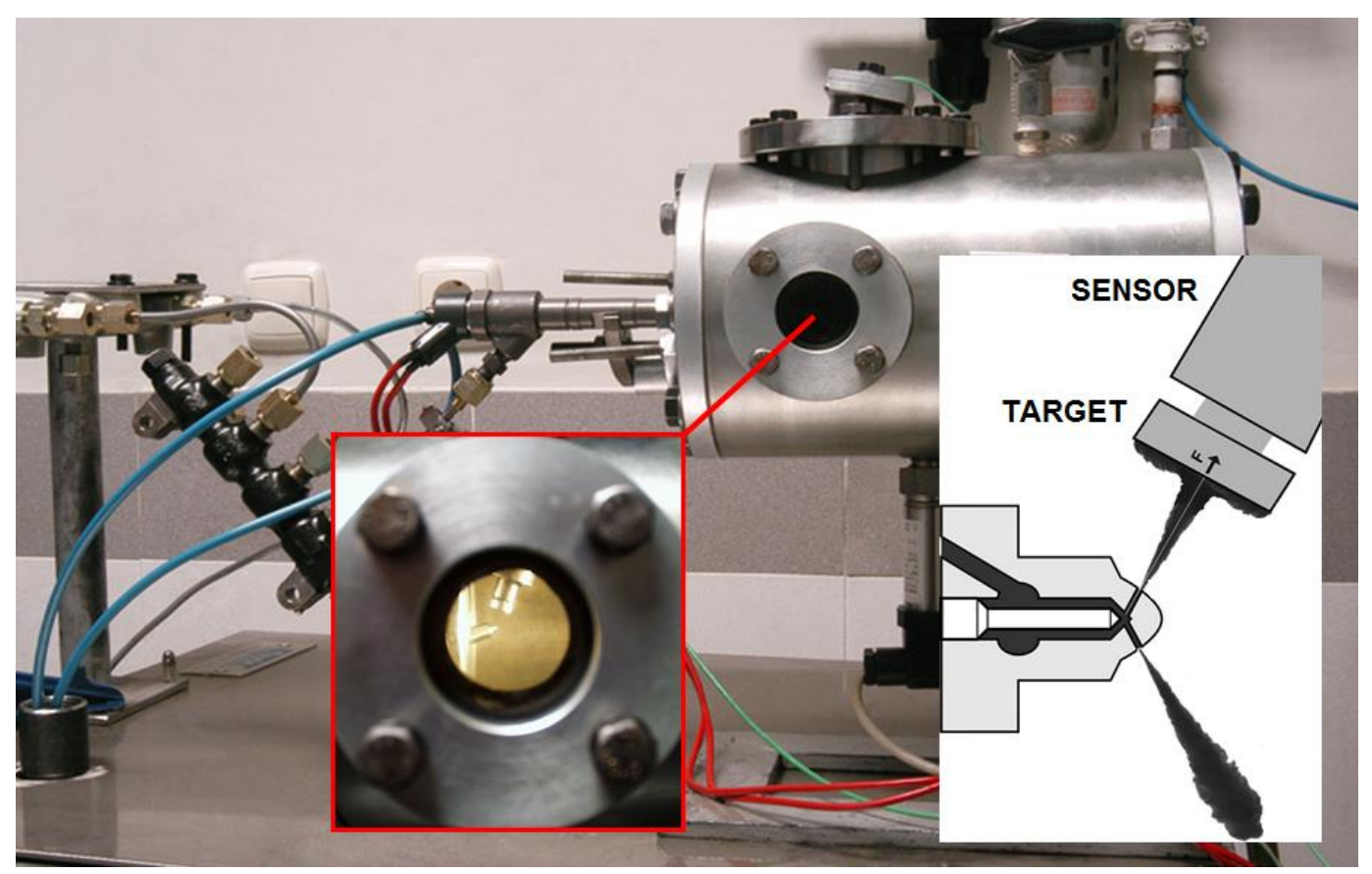

Figure 2: Momentum test rig. 

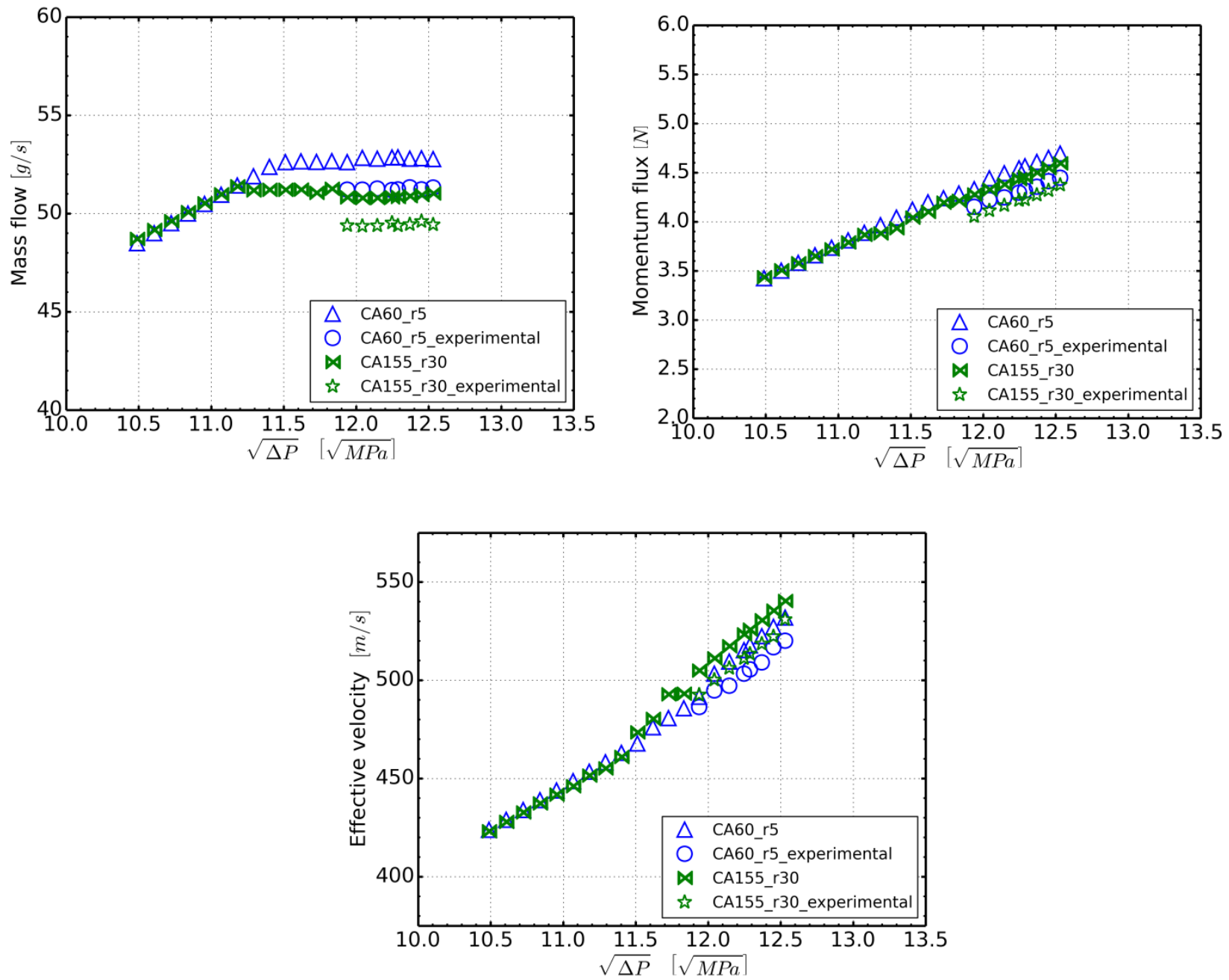

Figure 3: Model validation for inclination angle $60^{\circ}$ and $155^{\circ}$ at $160 \mathrm{MPa}$. 

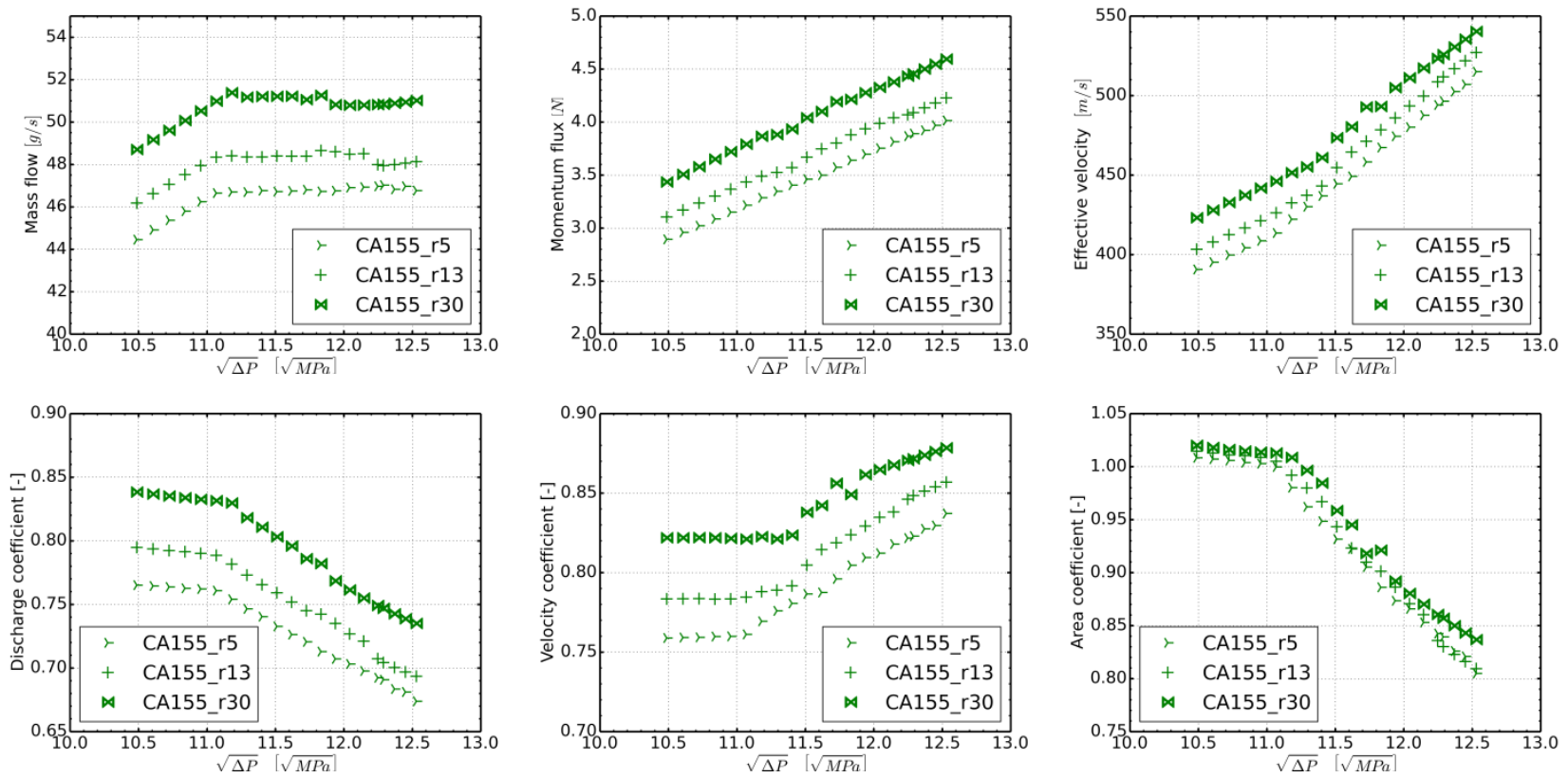

Figure 4: Mass flow rate, momentum flux and effective velocity for the nozzle in the upper part, and non-dimensional flux parameters in the lower part for the Nozzles CA155 at $160 \mathrm{MPa}$ of injection pressure and different backpressures.
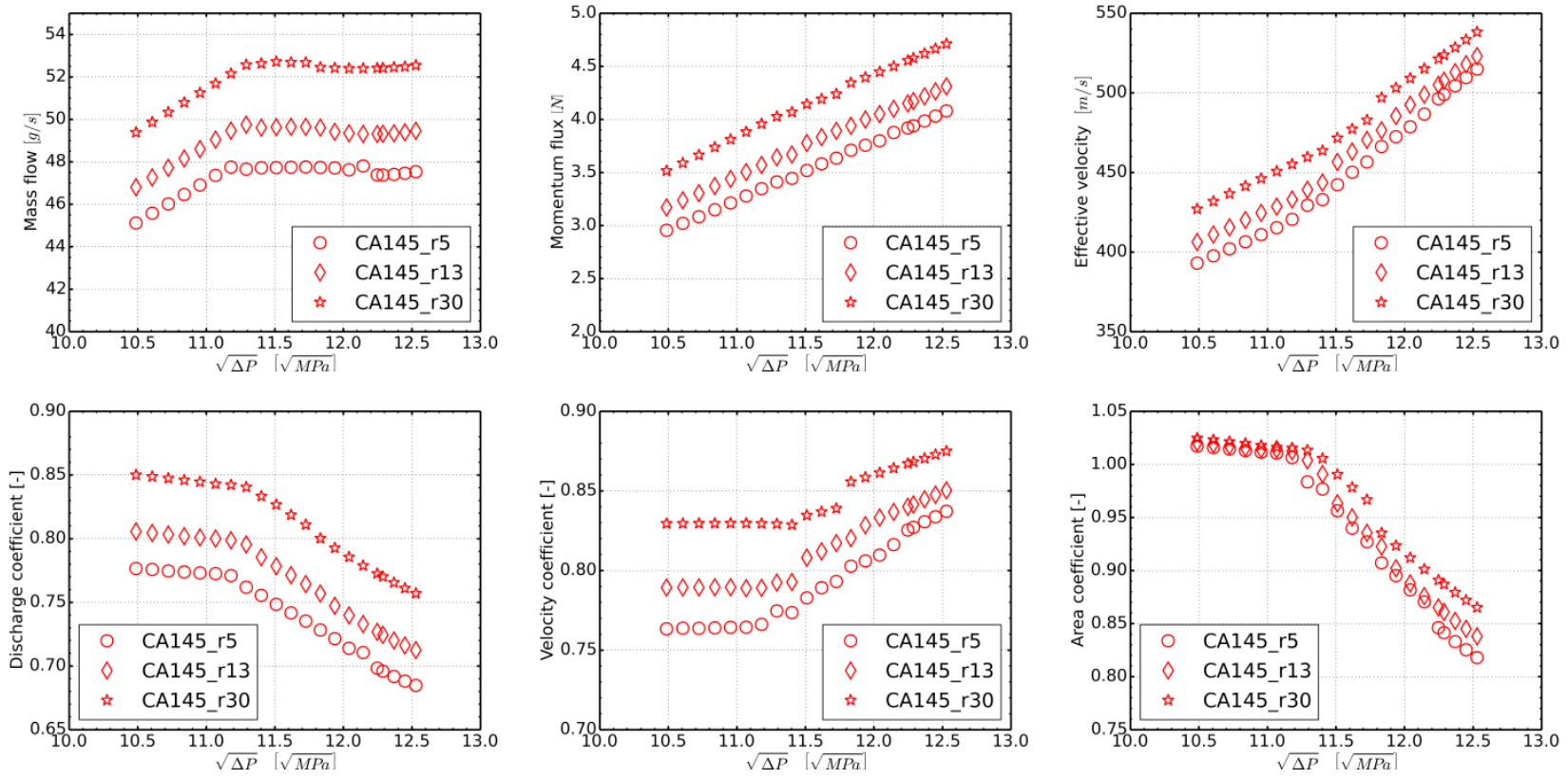

Figure 5: Mass flow rate, momentum flux and effective velocity for the nozzle in the upper part, 
and non-dimensional flux parameters in the lower part for the Nozzles CA145 at $160 \mathrm{MPa}$ of injection pressure and different backpressures.
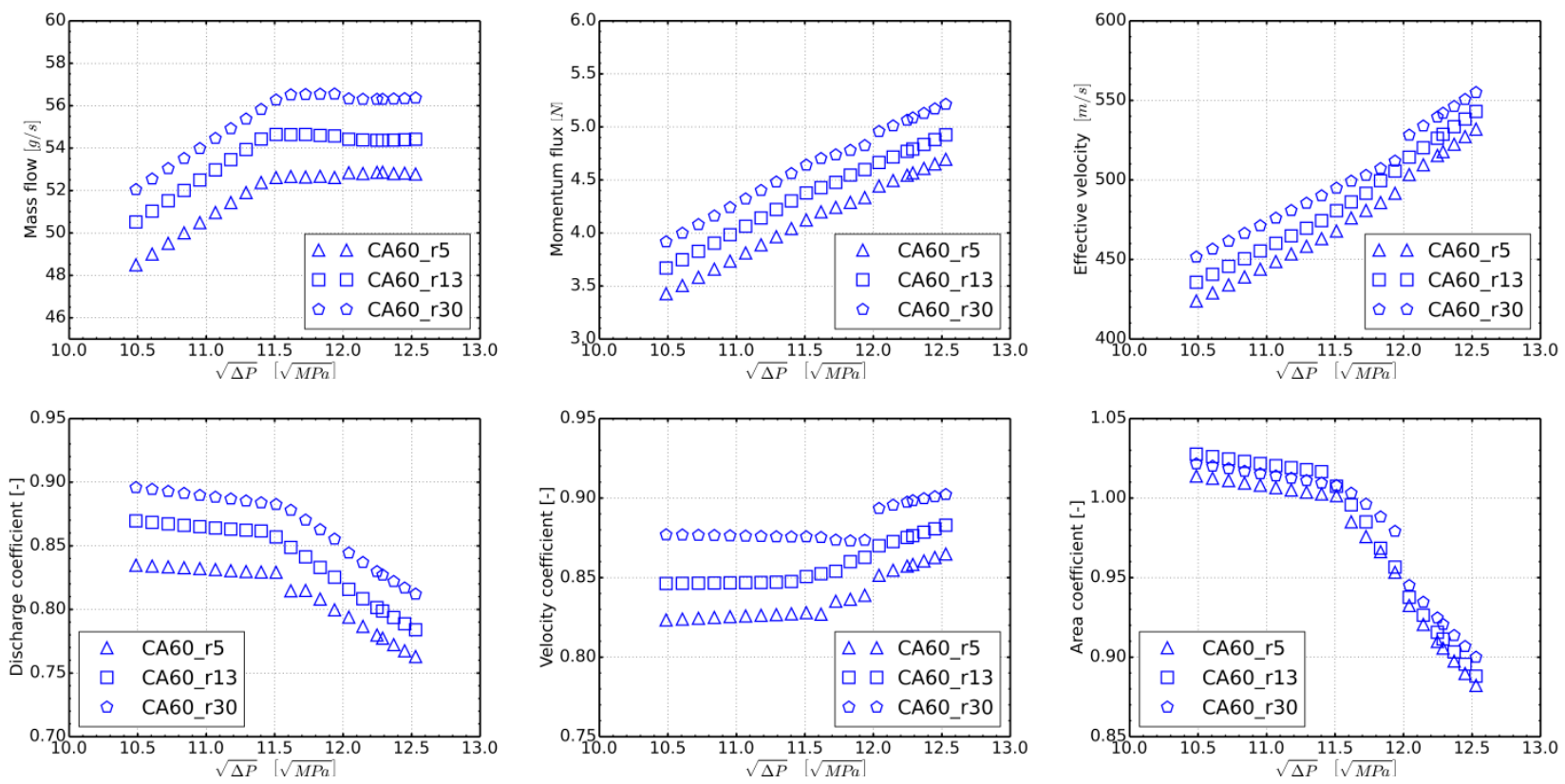

Figure 6: Mass flow rate, momentum flux and effective velocity for the nozzle in the upper part, and non-dimensional flux parameters in the lower part for the Nozzles CA60 at $160 \mathrm{MPa}$ of injection pressure and different backpressures. 


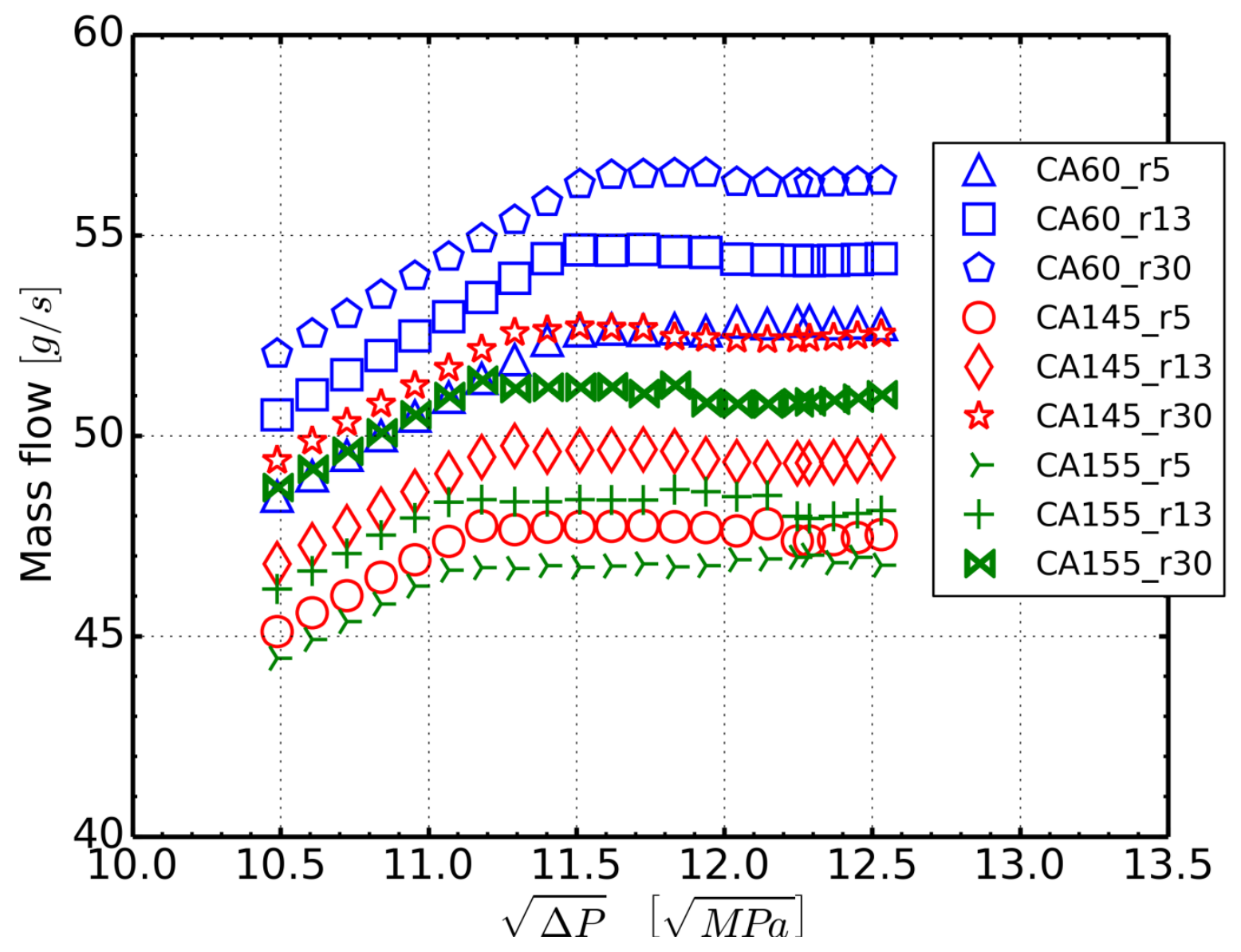

Figure 7: Comparison in terms of mass flow rate for all the nozzles. 


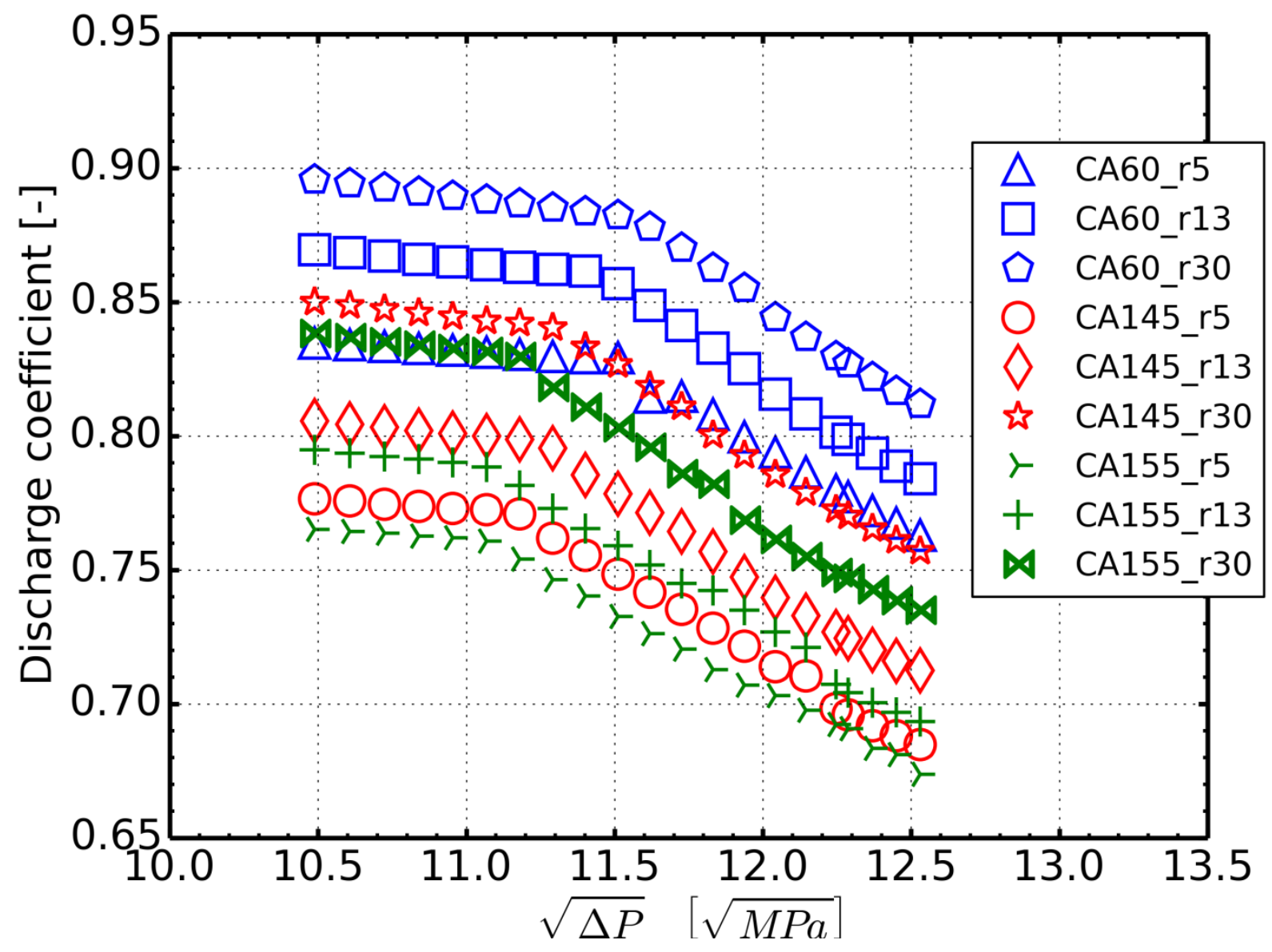

Figure 8: Comparison in terms of discharge coefficient for all the nozzles. 


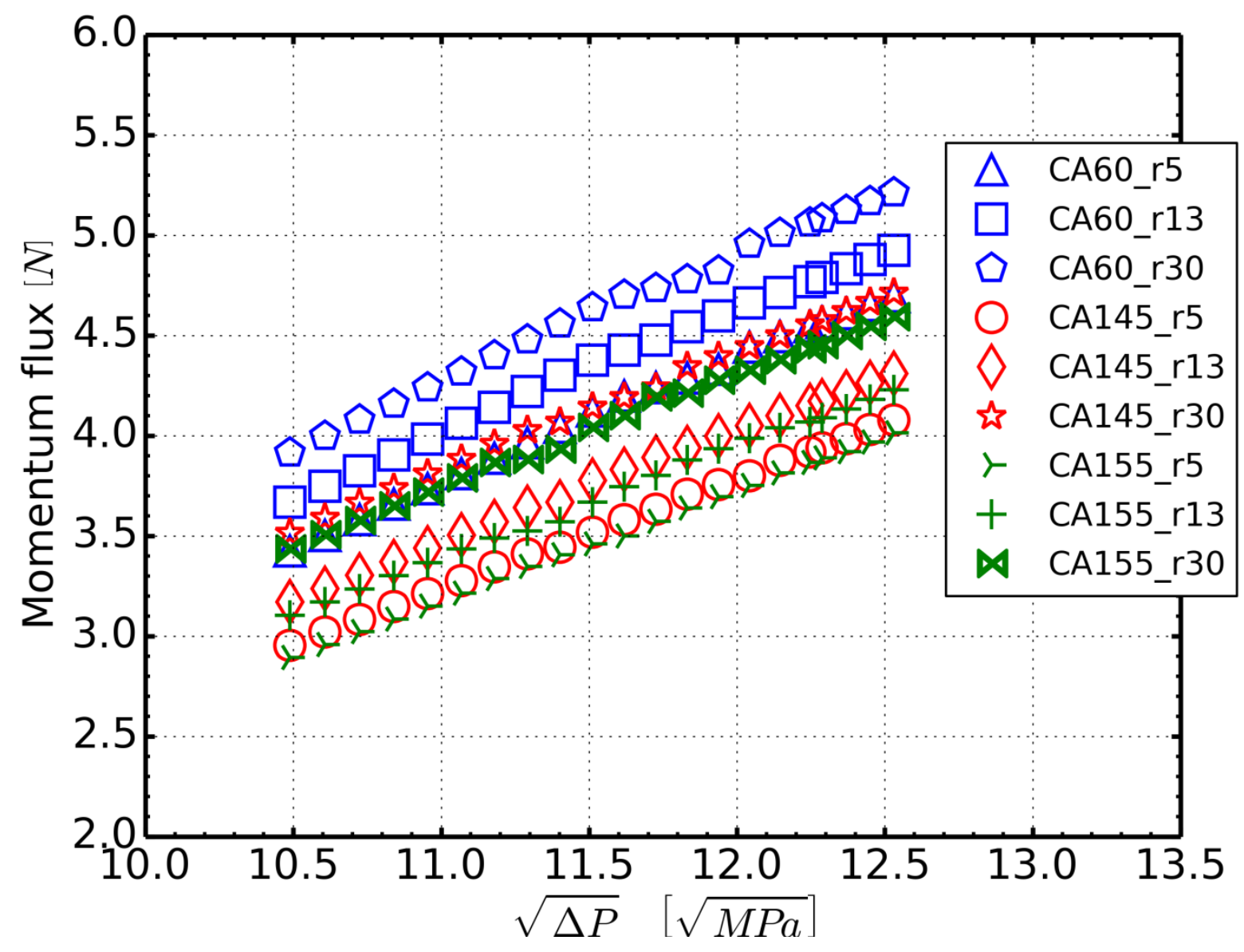

Figure 9: Comparison in terms of momentum flux for all the nozzles. 


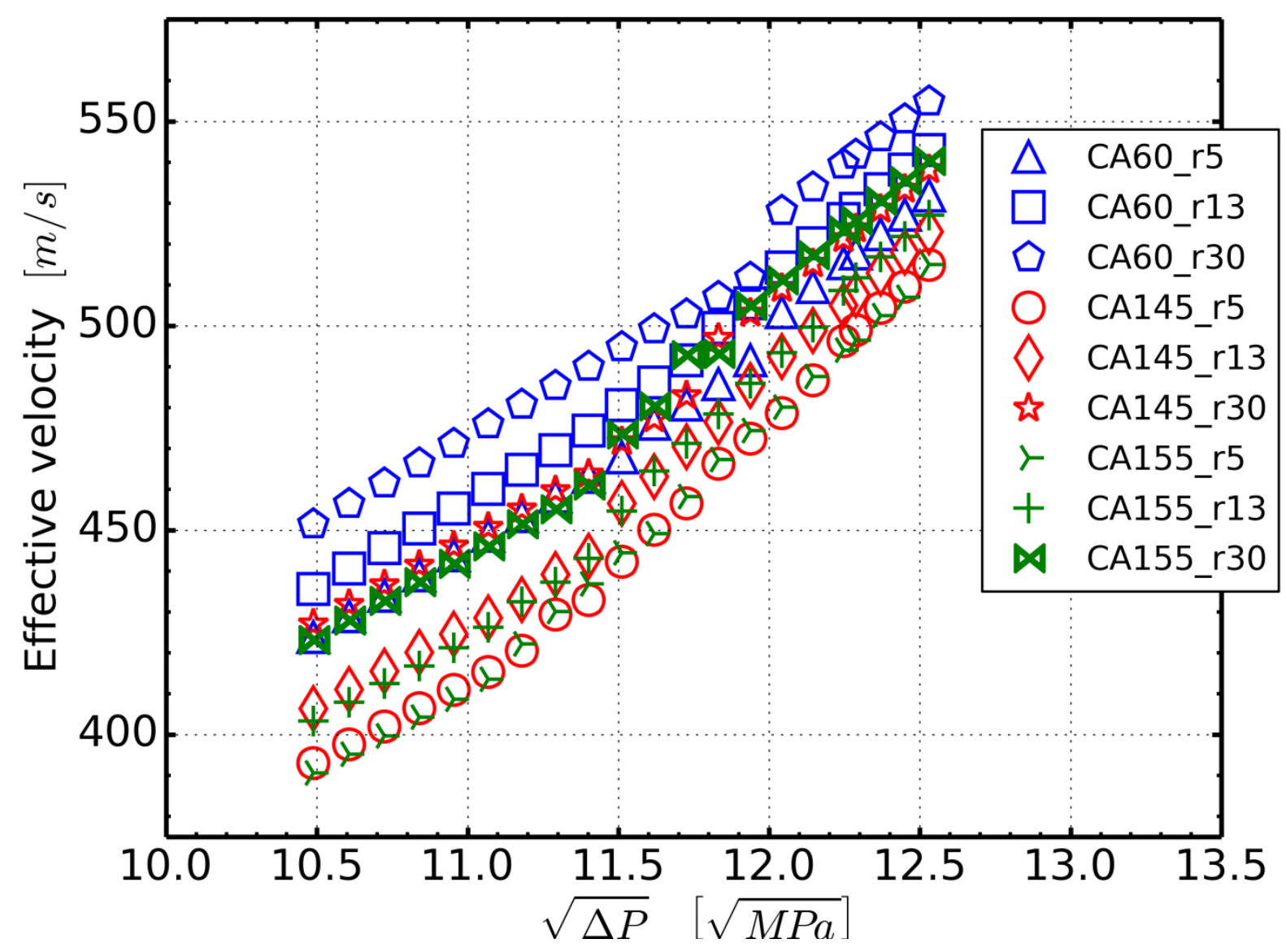

Figure 10: Comparison in terms of effective velocity for all the nozzles. 


\section{Discharge coefficient}

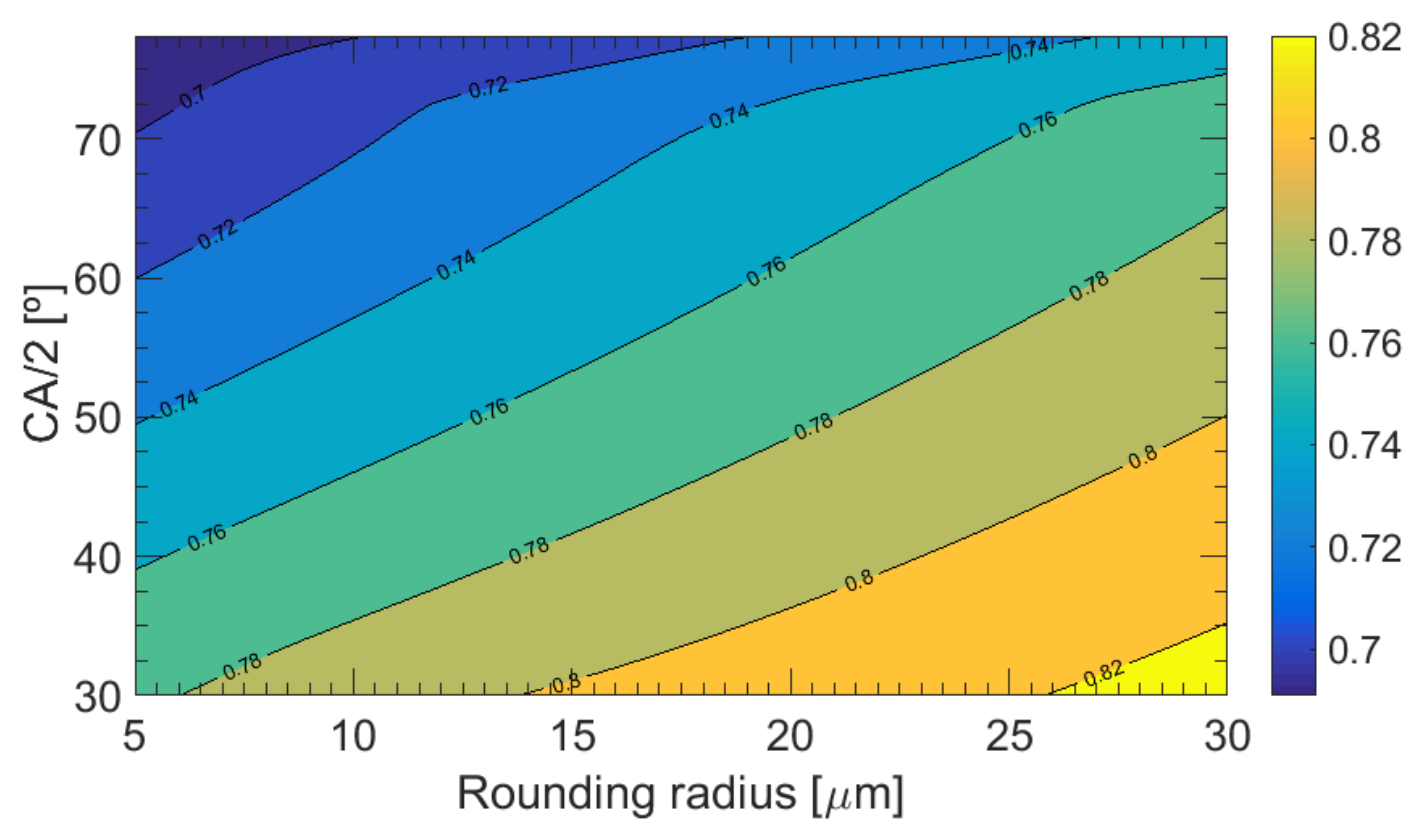

\section{Kcrit}

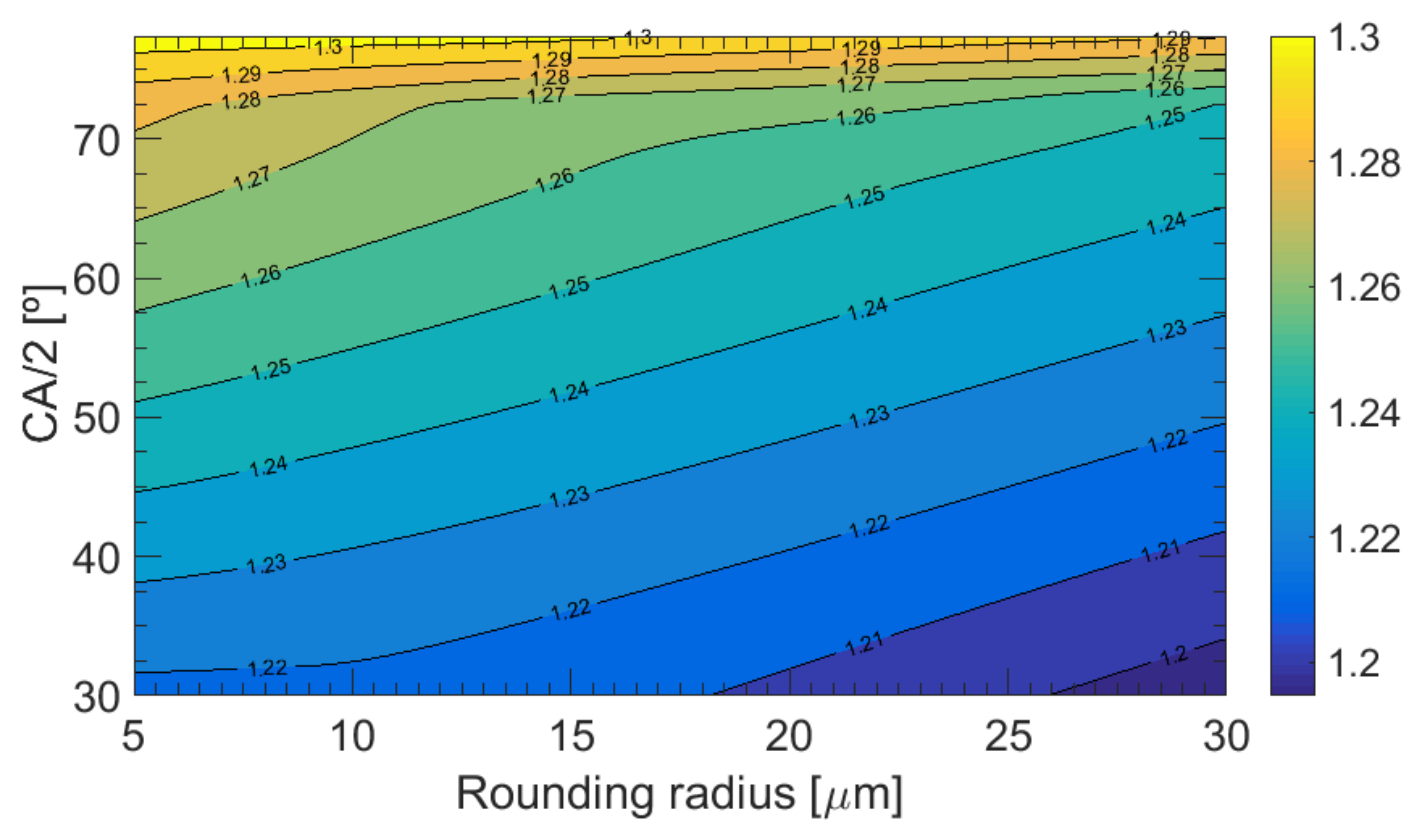

Figure 11: Discharge coefficient as a function of orifice angle (CA/2) and rounding radius for the injection condition $\mathrm{P}_{\text {inj }}=160 \mathrm{MPa}$ and $\mathrm{P}_{\text {back }}=9 \mathrm{MPa}$ (upper part). $\mathrm{K}_{\text {crit }}$ as a function of the half of the orifice angle $(\mathrm{CA} / 2)$ and the rounding radius (lower part). 


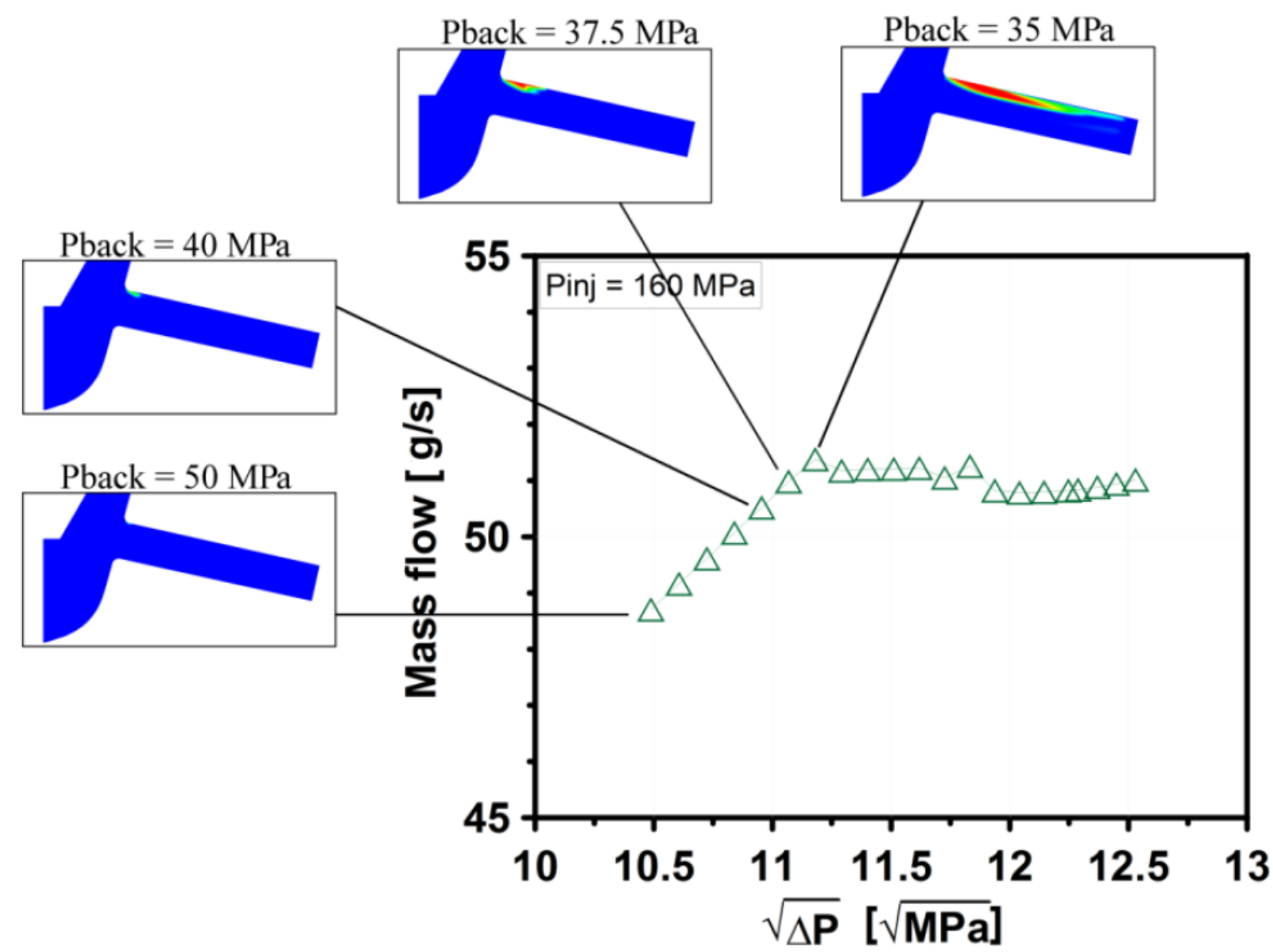

Figure 12: Vapour field and mass flow for the nozzle CA155 for the injection pressure of $160 \mathrm{MPa}$ and different backpressures. 


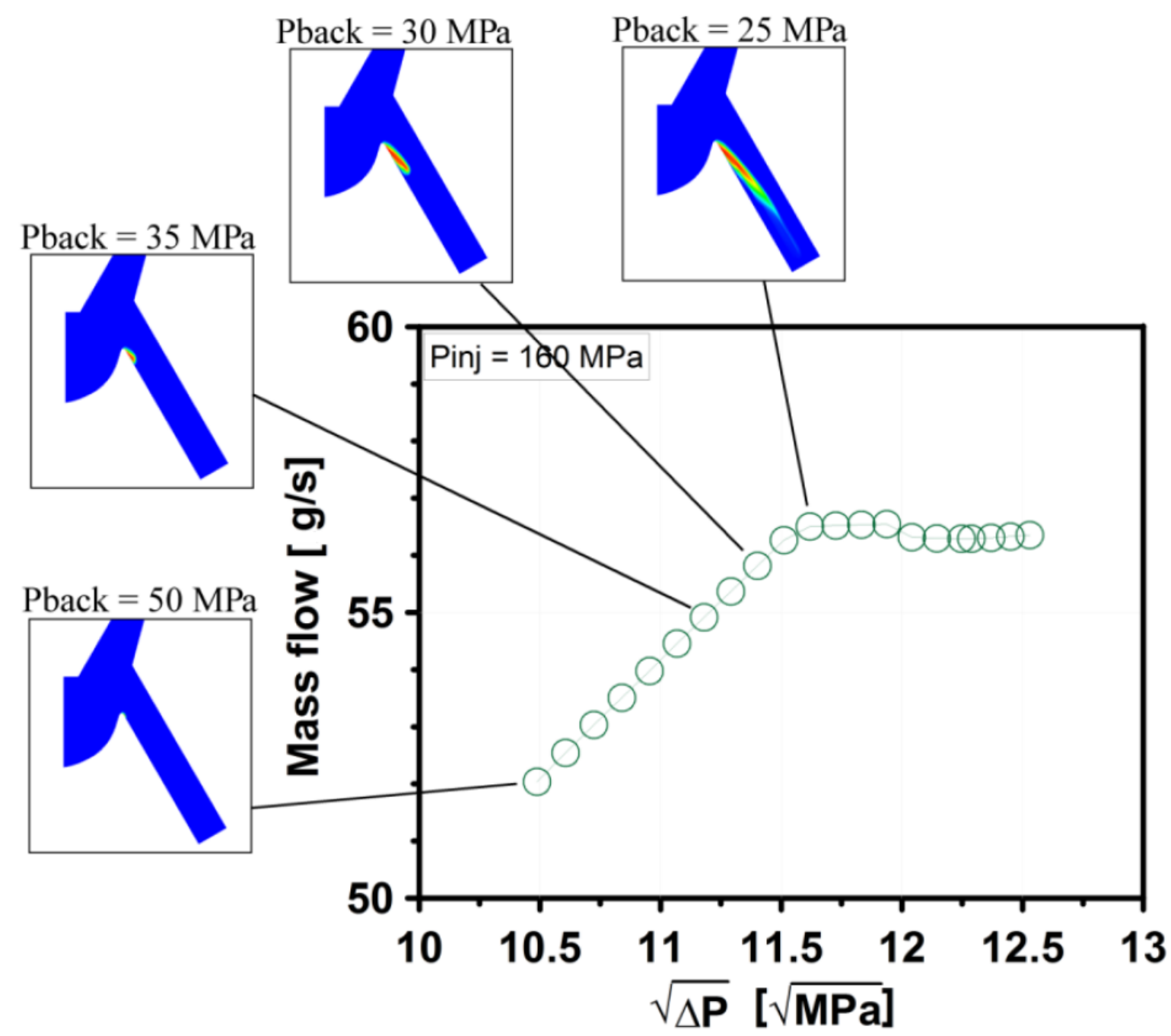

Figure 13: Vapour field and mass flow for the nozzle CA60 for the injection pressure of $160 \mathrm{MPa}$ and different backpressures. 


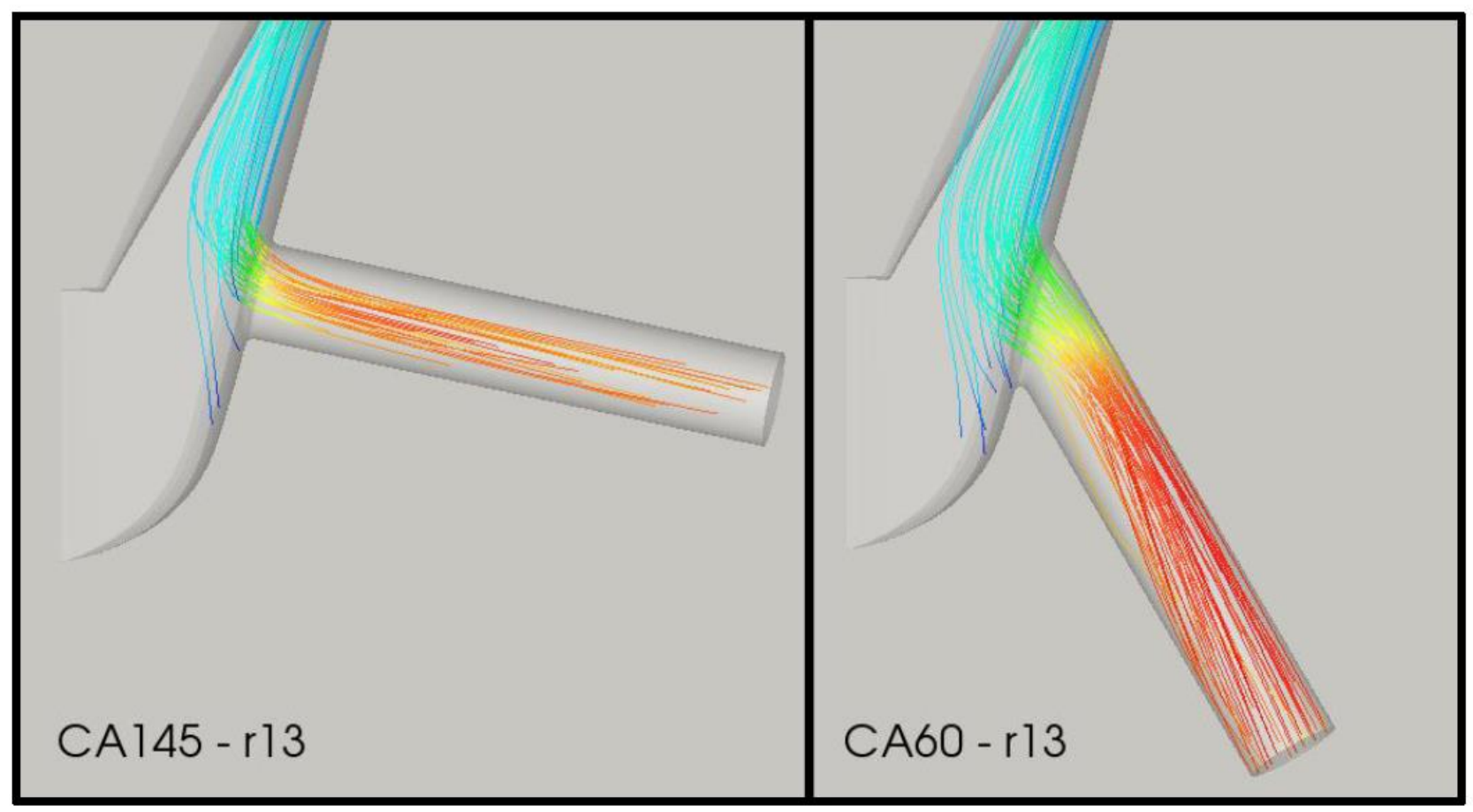

Figure 14: Differences in streamlines between nozzles CA145-r13 and CA60-r13 